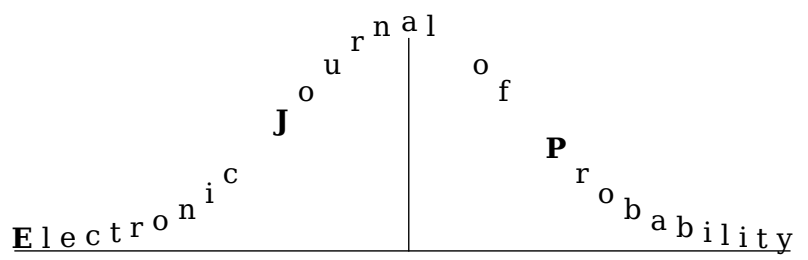

Electron. J. Probab. 26 (2021), article no. 17, 1-25.

ISSN: 1083-6489 https://doi.org/10.1214/21-EJP589

\title{
Multiple points of Gaussian random fields *
}

\author{
Robert C. Dalang ${ }^{\dagger} \quad$ Cheuk Yin Lee ${ }^{\ddagger} \quad$ Carl Mueller $^{\S} \quad$ Yimin Xiao ${ }^{\Uparrow}$
}

\begin{abstract}
This paper is concerned with the existence of multiple points of Gaussian random fields. Under the framework of Dalang et al. (2017), we prove that, for a wide class of Gaussian random fields, multiple points do not exist in critical dimensions. The result is applicable to fractional Brownian sheets and the solutions of systems of stochastic heat and wave equations.
\end{abstract}

Keywords: multiple points; Gaussian random fields; critical dimension; fractional Brownian sheet; stochastic heat and wave equations.

MSC2020 subject classifications: 60G15; 60G17; 60G60.

Submitted to EJP on October 30, 2019, final version accepted on January 30, 2021.

\section{Introduction}

Let $v=\left\{v(x), x \in \mathbb{R}^{k}\right\}$ be a centered continuous $\mathbb{R}^{d}$-valued Gaussian random field defined on a probability space $(\Omega, \mathscr{F}, \mathrm{P})$ with i.i.d. components. Write $v(x)=$ $\left(v_{1}(x), \ldots, v_{d}(x)\right)$ for $x \in \mathbb{R}^{k}$. For a set $T \subset \mathbb{R}^{k}$ (e.g., $T=(0, \infty)^{k}$, or $\left.T=[0,1]^{k}\right)$ and an integer $m \geq 2$, we say that $z \in \mathbb{R}^{d}$ is an $m$-multiple point of $v(x)$ on $T$ if, with positive probability, there are $m$ distinct points $x^{1}, \ldots, x^{m} \in T$ such that $z=v\left(x^{1}\right)=\cdots=v\left(x^{m}\right)$.

Several authors have studied the existence of multiple points of Gaussian random fields. Sufficient conditions or necessary conditions for the case of a fractional Brownian motion $B^{H}=\left\{B^{H}(t), t \in \mathbb{R}^{k}\right\}$ in $\mathbb{R}^{d}$ were proved by Kôno [8], Goldman [6], Rosen [12]. Their results show that if $k m>(m-1) H d$ then $B^{H}$ has $m$-multiple points on any interval

*The research of R.C. Dalang is partially supported by the Swiss National Foundation for Scientific Research, the research of C. Mueller is partially supported by a Simons grant, and Y. Xiao is partially supported by NSF grants DMS-1607089 and DMS-1855185.

${ }^{\dagger}$ Institut de mathématiques, École Polytechnique Fédérale de Lausanne, Station 8, CH-1015 Lausanne, Switzerland. E-mail: robert.dalang@epfl.ch

${ }^{\ddagger}$ Department of Statistics and Probability, Michigan State University, East Lansing, MI 48824, United States. Current address: Institut de mathématiques, École Polytechnique Fédérale de Lausanne, Station 8, CH-1015 Lausanne, Switzerland. E-mail: cheuk. lee@epfl.ch

${ }^{\S}$ Department of Mathematics, University of Rochester, Rochester, NY 14627, United States.

E-mail: carl.e.muellerarochester.edu

"Department of Statistics and Probability, Michigan State University, East Lansing, MI 48824, United States.

E-mail: xiao@stt.msu.edu 
$T \subseteq \mathbb{R}^{k}$; and if $k m<(m-1) H d$ then $B^{H}$ has no $m$-multiple points on $\mathbb{R}^{k} \backslash\{0\}$. Rosen [12] also considered the existence of multiple points of the Brownian sheet by studying its self-intersection local times.

In the critical dimensions (i.e., $k m=(m-1) H d$ for $B^{H}$ ), the problem for proving the non-existence of multiple points is more difficult. For fractional Brownian motion and the Brownian sheet, the problem was resolved by Talagrand [13] and by Dalang et al. [3] and Dalang and Mueller [4], respectively. The methods in [13] and [3, 4] are different.

Our research in this paper is motivated by the interest in studying the intersection problems for the solutions of systems of stochastic heat and wave equations with constant coefficients, where the method in $[3,4]$ fails in general. Our main purpose is to continue the work of [5] and extend Talagrand's approach in [13] to a large class of Gaussian random fields which include fractional Brownian sheets and the solutions of systems of stochastic heat and wave equations with constant coefficients. As a byproduct, our theorem provides an alternative proof for the results in $[3,4]$ by using general Gaussian principles and the harmonizable representation of the Brownian sheet.

The result of this paper relies on a covering argument originated by Talagrand [13], and later further developed by Dalang et al. [5]. In this paper, the main ingredient for the covering argument is Proposition 3.6, which states that for any distinct points $s^{1}, \ldots, s^{m} \in T$, with high probability, there are small neighbourhoods of $s^{i}$ in which the maximum of the increments $v\left(x^{i}\right)-v\left(s^{i}\right)(1 \leq i \leq m)$ could be smaller than one would expect from the Hölder regularity. This observation allows us to use balls of different radii to construct an efficient random cover for the set of multiple points, which is essential for proving the non-existence of multiple points in the critical dimension.

The paper is organized as follows. In Section 2, we state our assumptions and the main result of this paper, Theorem 2.5. In Section 3, we establish some necessary ingredients for proving Theorem 2.5 and, in Section 4, we prove the main theorem. In Section 5, we provide several examples of Gaussian random fields to which the theorem can be applied. These examples include the Brownian sheet, fractional Brownian sheets, and the solutions of systems of stochastic heat and wave equations.

Throughout the article, we use $K$ or $c$ to denote a constant that may vary at each occurrence. Specific constants will be denoted by $K_{1}, K_{2}, c_{1}$, etc.

\section{Assumptions and the main result}

By a compact interval (or rectangle) in $\mathbb{R}^{k}$ we mean a set $I$ of the form $\prod_{j=1}^{k}\left[c_{j}, d_{j}\right]$, where $c_{j}<d_{j}$. Throughout this paper, we assume that $T \subset \mathbb{R}^{k}$ is a fixed index set that can be written as a countable union of compact intervals. To avoid triviality in studying the multiple points of $\left\{v(x), x \in \mathbb{R}^{k}\right\}$, one may take, for example, $T=\mathbb{R}^{k} \backslash\{0\}$ or $T=(0, \infty)^{k}$.

In the following, Assumption 2.1 is a slightly simplified reformulation of Assumption 2.1 in [5] and Assumption 2.2 below is a reinforced version of Assumption 2.4 in [5].

Assumption 2.1. There exists a centered Gaussian random field $\left\{v(A, x), A \in \mathscr{B}\left(\mathbb{R}_{+}\right), x \in\right.$ $T\}$, where $\mathscr{B}\left(\mathbb{R}_{+}\right)$is the Borel $\sigma$-algebra on $\mathbb{R}_{+}=[0, \infty)$, such that the following hold:

(a) For all $x \in T, A \mapsto v(A, x)$ is an $\mathbb{R}^{d}$-valued white noise (or, more generally, an independently scattered Gaussian noise with a control measure $\mu$ ) with i.i.d. components, $v\left(\mathbb{R}_{+}, x\right)=v(x)$, and $v(A, \cdot)$ and $v(B, \cdot)$ are independent whenever $A$ and $B$ are disjoint.

(b) There exist constants $\gamma_{j}>0, j=1, \ldots, k$ with the following properties: For every compact interval $F \subset T$, there exist constants $c_{0}>0$ and $a_{0} \geq 0$ such that for all 
$a_{0} \leq a<b \leq \infty$ and $x, y \in F$,

$$
\|v([a, b), x)-v(x)-v([a, b), y)+v(y)\|_{L^{2}} \leq c_{0}\left(\sum_{j=1}^{k} a^{\gamma_{j}}\left|x_{j}-y_{j}\right|+b^{-1}\right)
$$

and

$$
\left\|v\left(\left[0, a_{0}\right), x\right)-v\left(\left[0, a_{0}\right), y\right)\right\|_{L^{2}} \leq c_{0} \sum_{j=1}^{k}\left|x_{j}-y_{j}\right|
$$

In the above, $\|X\|_{L^{2}}=\left(\mathrm{E}|X|^{2}\right)^{1 / 2}$ for a random vector $X$, where $|X|$ is the Euclidean norm of $X$.

Notice that in Assumption 2.1 the constants $a_{0}$ and $c_{0}$ may depend on $F$, but $\gamma_{j}$ $(j=1, \ldots, k)$ do not. As shown by Dalang et al. [5], the parameters $\gamma_{j}(j=1, \ldots, k)$ play important roles in characterizing sample path properties (e.g., regularity, fractal properties, hitting probabilities) of the random field $\{v(x), x \in T\}$.

Let $\alpha_{j}=\left(\gamma_{j}+1\right)^{-1}$ and $Q=\sum_{j=1}^{k} \alpha_{j}^{-1}$. Define the metric $\Delta$ on $\mathbb{R}^{k}$ by

$$
\Delta(x, y)=\sum_{j=1}^{k}\left|x_{j}-y_{j}\right|^{\alpha_{j}}
$$

For $x \in T$ and $r>0$, denote by $S(x, r)=\left\{y \in \mathbb{R}^{k}: \Delta(x, y) \leq r\right\}$ the closed ball with center $x$ and radius $r$ in the metric $\Delta$ in (2.3) and let $B_{r}(x)=\prod_{j=1}^{k}\left[x_{j}-r^{1 / \alpha_{j}}, x_{j}+r^{1 / \alpha_{j}}\right]$. Notice that $S(x, r) \subseteq B_{r}(x)$ and $B_{r / k}(x) \subseteq S(x, r)$.

Assumption 2.2. For every compact interval $F \subset T$, there exist constants $0<\varepsilon_{0} \leq 1$, $c \geq 3, c^{\prime} \geq 2 c$ and $\delta_{j} \in\left(\alpha_{j}, 1\right], j=1, \ldots, k$, such that for every $0<\rho \leq \varepsilon_{0}$ there is a finite constant $C$ (which may depend on $F, \rho$ and $\delta_{j}$ ) and the following property holds:

For every $x \in F$ with $B_{c \rho}(x) \subset F$, there is $x^{\prime} \in B_{c \rho}(x)$ such that for every $i=1, \ldots, d$ the condition

$$
\left|\mathrm{E}\left(\left(v_{i}(y)-v_{i}(\bar{y})\right) v_{i}\left(x^{\prime}\right)\right)\right| \leq C \sum_{j=1}^{k}\left|y_{j}-\bar{y}_{j}\right|^{\delta_{j}}
$$

holds for all $y, \bar{y}$ in each of the following cases:

(i) $y, \bar{y} \in B_{2 \rho}(x)$;

(ii) $y, \bar{y} \in B_{2 \rho}(\tilde{x})$, for any $\tilde{x}$ such that $B_{2 \rho}(\tilde{x}) \subset F$ and $\Delta(x, \tilde{x}) \geq c^{\prime} \rho$.

Assumption 2.2 states that for every fixed point $x \in T$, one can find a reference point $x^{\prime}$ (which may depend on $x$ ) such that for all $y, \bar{y}$ that are either in a small neighborhood of $x$, or are sufficiently away from a neighborhood of $x$ (hence away from $x^{\prime}$ ), the covariances in (2.4) are smoother than what one gets from the Cauchy-Schwarz inequality and Lemma 3.1 below, which lead to $\alpha_{j}$ instead of $\delta_{j}$ in the exponents.

As an illustration example, let $\left\{v(x), x \in \mathbb{R}^{k}\right\}$ be a real-valued fractional Brownian motion with index $H \in(0,1)$ and let $F \subset \mathbb{R}^{k} \backslash\{0\}$ be a compact interval. Then for any $\rho>0$ and $x^{\prime}, y, \bar{y} \in F$ that satisfy $\min \left\{|y|,|\bar{y}|,\left|y-x^{\prime}\right|,\left|\bar{y}-x^{\prime}\right|\right\} \geq \rho$, where $|\cdot|$ is the Euclidean norm in $\mathbb{R}^{k}$, we use the mean value theorem to derive

$$
\begin{aligned}
\left|\mathrm{E}\left((v(y)-v(\bar{y})) v_{i}\left(x^{\prime}\right)\right)\right| & =\frac{1}{2}\left(|y|^{2 H}-|\bar{y}|^{2 H}-\left|y-x^{\prime}\right|^{2 H}+\left|\bar{y}-x^{\prime}\right|^{2 H}\right) \\
& \leq C|y-\bar{y}|,
\end{aligned}
$$


where $C$ is a constant depending on $H, \rho$ (if $H \leq 1 / 2$ ) and the compactness of $F$ (if $H>1 / 2$ ). Hence Assumption 2.2 holds with $c=3, c^{\prime}=6$ and $\delta_{j}=1$ for $j=1, \ldots, k$. This was observed in Lemma 3.2 of Talagrand [13]. As we will see in Section 5, verifications of Assumption 2.2 for fractional Brownian sheets and the solutions to stochastic heat and wave equations are more involved.

Now we introduce an additional non-degeneracy assumption.

Assumption 2.3. For any $m$ distinct points $x^{1}, \ldots, x^{m} \in T, v_{1}\left(x^{1}\right), \ldots, v_{1}\left(x^{m}\right)$ are linearly independent random variables, or equivalently, the Gaussian distribution of the vector $\left(v_{1}\left(x^{1}\right), \ldots, v_{1}\left(x^{m}\right)\right)$ is non-degenerate.

Remark 2.4. Assumption 2.3 is also equivalent to $\operatorname{Var}\left(v_{1}\left(x^{1}\right)\right)>0$ and, for every $\ell=$ $2, \ldots, m$, the conditional variance of $v_{1}\left(x^{\ell}\right)$ given $v_{1}\left(x^{j}\right), j \leq \ell-1$, is positive.

The main result of this paper is the following.

Theorem 2.5. Let $m \geq 2$. Suppose that Assumptions 2.1, 2.2 and 2.3 hold. If $m Q \leq$ $(m-1) d$, then $\{v(x), x \in T\}$ has no $m$-multiple points almost surely.

\section{Preliminaries}

In this section, we provide some preliminaries that will be used for proving Theorem 2.5. Clearly it suffices to prove that if $m Q \leq(m-1) d$ then, for every compact interval $F \subset T,\{v(x), x \in F\}$ has no $m$-multiple points almost surely. Hence, without loss of generality, we assume in Sections 3 and 4 that $T$ is a compact interval.

Fix an integer $m \geq 2$. For an integer $n \geq 1$, let $A_{n}$ be the countable subset of $T^{m}$ defined by

$$
A_{n}=\left\{\left(t^{1}, \ldots, t^{m}\right): t^{i} \in T \cap \mathbb{Q}^{k}, \Delta\left(t^{i}, t^{j}\right) \geq 1 / n \text { for } i \neq j\right\} .
$$

Since $T$ is compact, we see that the closure $\bar{A}_{n}$ is compact. Moreover, $\bigcup_{n=1}^{\infty} A_{n}$ is dense in $T^{m}$.

For any constant $\rho>0$, let $B_{\rho}^{i}=B_{\rho}\left(t^{i}\right)(i=1, \ldots, m)$. It is clear that if $\left(t^{1}, \ldots, t^{m}\right) \in$ $A_{n}$ and $\rho \in(0,1 / n)$ is small enough, then the intervals $B_{\rho}^{i}(i=1, \ldots, m)$ are disjoint.

Consider the random set

$$
\begin{aligned}
M_{t^{1}, \ldots, t^{m} ; \rho}=\left\{z \in \mathbb{R}^{d}:\right. & \exists\left(x^{1}, \ldots, x^{m}\right) \in \prod_{i=1}^{m} B_{\rho}^{i} \\
& \text { such that } \left.z=v\left(x^{1}\right)=\cdots=v\left(x^{m}\right)\right\},
\end{aligned}
$$

which is the intersection of the images $v\left(B_{\rho}^{i}\right)$ for $i=1, \ldots, m$ and is contained in the set of $m$-multiple points. On the other hand, let $z \in \mathbb{R}^{d}$ be an $m$-multiple point, i.e., there are $m$ distinct points $x^{1}, \ldots, x^{m} \in T$ such that $z=v\left(x^{1}\right)=\cdots=v\left(x^{m}\right)$. Then for $n$ large enough we have $\Delta\left(x^{i}, x^{j}\right)>3 / n$ for $i \neq j$. It follows from the triangle inequality that, for any constant $\rho \in(0,1 / n)$, there is $\left(t^{1}, \ldots, t^{m}\right) \in A_{n}$ such that $\Delta\left(x^{i}, t^{i}\right)<\rho$. This implies that $z \in M_{t^{1}, \ldots, t^{m} ; \rho}$. Thus we have verified that the set of $m$-multiple points of $\{v(x): x \in T\}$ can be written as a countable union

$$
\bigcup_{n \geq 1} \bigcap_{\rho \in(0,1 / n) \cap \mathbb{Q}} \bigcup_{\left(t^{1}, \ldots, t^{m}\right) \in A_{n}} M_{t^{1}, \ldots, t^{m} ; \rho} .
$$

We will use this observation in Section 4 to complete the proof of Theorem 2.5.

For the rest of this section, we fix an integer $n \geq 1$. Let $\rho_{0} \in(0,1 / n)$ be the small constant given in Lemma 3.8 below. Notice that the constant $\rho_{0}$ is independent of $\left(t^{1}, \ldots, t^{m}\right) \in A_{n}$. For simplicity of notation, we assume that $B_{\rho_{0}}\left(t^{i}\right) \subseteq T$ for $i=1, \ldots, m$ (otherwise we take the intersection with $T$ ), and we omit the subscripts $t^{1}, \ldots, t^{m}$ in (3.2) and write $M_{t^{1}, \ldots, t^{m} ; \rho}$, as $M_{\rho}$. 
Recall from [5] that, under Assumption 2.1, $\Delta$ provides an upper bound for the $L^{2}$-norm of the increments of $\{v(x), x \in T\}$ and in particular $v(x)$ is continuous in $L^{2}(\Omega, \mathscr{F}, \mathrm{P})$.

Lemma 3.1. [5, Proposition 2.2] Under Assumption 2.1, for all $x, y \in T$ with $\Delta(x, y) \leq$ $\min \left\{a_{0}^{-1}, 1\right\}$, we have $\|v(x)-v(y)\|_{L^{2}} \leq 4 c_{0} \Delta(x, y)$.

Assumption 2.1 suggests that for any $s \in T$ and $x$ that is close to $s$, the increment $v(x)-v(s)$ can be approximated well by $v([a, b), x)-v([a, b), s)$ if we choose $a$ and $b$ carefully. The following lemma from [5] quantifies the approximation error on $S(s, c r)$.

Lemma 3.2. Let $c>0$ be a constant. Consider $b>a>1, \varepsilon_{1}>r>0$, where $\varepsilon_{1}>0$ is a small constant. Set

$$
A=\sum_{j=1}^{k} a^{\alpha_{j}^{-1}-1} r^{\alpha_{j}^{-1}}+b^{-1}
$$

There are constants $A_{0}, \tilde{K}$ and $\tilde{c}$ (depending on $c_{0}$ in Assumption 2.1 and $c$ ) such that if $A \leq A_{0} r$ and

$$
u \geq \tilde{K} A \log ^{1 / 2}\left(\frac{r}{A}\right)
$$

then for any $s \in T$,

$$
\mathrm{P}\left\{\sup _{x \in S(s, c r)}|v(x)-v(s)-(v([a, b), x)-v([a, b), s))| \geq u\right\} \leq \exp \left(-\frac{u^{2}}{\tilde{c} A^{2}}\right) .
$$

Remark 3.3. The constant $c$ in Lemma 3.2 and Proposition 3.6 below is not important. It merely helps to simplify the presentation in Section 4, where sometimes we switch back and forth between a ball $S(s, r)$ and an interval $B_{r}(x)$.

For describing the contribution of the main part $v([a, b), x)-v([a, b), s)$, we will apply the small ball probability estimate given in Lemma 3.5 below. We refer to Lemma 2.2 of [14] for a general lower bound on the small ball probability of Gaussian processes. However, it was pointed out by Slobodan Krstic (personal communication) that the condition of that lemma is not correctly stated. Indeed, the lemma fails if we consider $S$ consisting of two points, and independent standard normal random variables indexed by the two points. We will make use of the following reformulation of the presentation of Talagrand's lower bound given by Ledoux [9, (7.11)-(7.13) on p. 257].

Lemma 3.4. Let $\{X(t), t \in S\}$ be a separable, $\mathbb{R}^{d}$-valued, centered Gaussian process indexed by a bounded set $S$ with the canonical metric $d_{X}(s, t)=\left(\mathrm{E}|X(s)-X(t)|^{2}\right)^{1 / 2}$. Let $N_{\varepsilon}(S)$ denote the smallest number of $d_{X}$-balls of radius $\varepsilon$ needed to cover $S$. If there is a decreasing function $\psi:(0, \delta] \rightarrow(0, \infty)$ such that $N_{\varepsilon}(S) \leq \psi(\varepsilon)$ for all $\varepsilon \in(0, \delta]$ and there are constants $c_{2} \geq c_{1}>1$ such that

$$
c_{1} \psi(\varepsilon) \leq \psi(\varepsilon / 2) \leq c_{2} \psi(\varepsilon)
$$

for all $\varepsilon \in(0, \delta]$, then there is a constant $K$ depending only on $c_{1}, c_{2}$ and $d$ such that for all $u \in(0, \delta)$,

$$
\mathrm{P}\left(\sup _{s, t \in S}|X(s)-X(t)| \leq u\right) \geq \exp (-K \psi(u)) .
$$

Let $\rho \in\left(0, \rho_{0} / 3\right)$ be a constant and let $\left(t^{1}, \ldots, t^{m}\right) \in A_{n}$. Recall that $B_{2 \rho}^{1}, \ldots, B_{2 \rho}^{m}$ are the rectangles centered at $t^{1}, \ldots, t^{m}$. By applying Assumption 2.1 and Lemma 3.4, we derive the following lemma.

Lemma 3.5. Suppose that Assumption 2.1 holds and $\rho \in\left(0, \rho_{0} / 3\right)$ is a constant. Then there exist constants $K$ and $0<\eta_{0}<\rho_{0} / 3$, depending on $c_{0}$ in Assumption 2.1, such that 
for all $\left(s^{1}, \ldots, s^{m}\right) \in B_{2 \rho}^{1} \times \cdots \times B_{2 \rho}^{m}$, for all $0<a<b$ and $0<u<r<\eta_{0}$, we have

$$
\mathrm{P}\left(\sup _{1 \leq i \leq m} \sup _{x^{i} \in S\left(s^{i}, r\right)}\left|v\left([a, b), x^{i}\right)-v\left([a, b), s^{i}\right)\right| \leq u\right) \geq \exp \left(-K \frac{r^{Q}}{u^{Q}}\right),
$$

where $Q=\sum_{j=1}^{k} \alpha_{j}^{-1}$.

Proof. As suggested by the proof of (3.3) in Talagrand [13], (3.7) can be derived from Lemma 3.4. However, there was a typo in the exponent in (3.3) in [13] (the ratio $\frac{r}{u^{1 / \alpha}}$ there should be raised to the power $N$ ) and the suggested proof by introducing the auxiliary process $Z$ does not give the correct power for $\frac{r}{u^{1 / \alpha}}$ in (3.3) in [13], which is needed for proving Proposition 3.4 in [13]. Hence we give a proof of (3.7).

For $\left(s^{1}, \ldots, s^{m}\right) \in B_{2 \rho}^{1} \times \cdots \times B_{2 \rho}^{m}$ and $r<\rho_{0} / 3$, define $S=\bigcup_{i=1}^{m} S\left(s^{i}, r\right)$. Under our assumption, we have $S\left(s^{i}, r\right) \subseteq T$ for $i=1, \ldots, m$. Thus, $S \subseteq T$. It follows from Assumption 2.1 that for all $x, y \in S$,

$$
\begin{aligned}
\|v([a, b), x)-v([a, b), y)\|_{L^{2}}^{2} & =\|v(x)-v(y)\|_{L^{2}}^{2}-\left\|v\left(\mathbb{R}_{+} \backslash[a, b), x\right)-v\left(\mathbb{R}_{+} \backslash[a, b), y\right)\right\|_{L^{2}}^{2} \\
& \leq\|v(x)-v(y)\|_{L^{2}}^{2}
\end{aligned}
$$

By Lemma 3.1, we have that the canonical metric for $\{v([a, b), x), x \in S\}$ satisfies

$$
d_{v}(s, t):=\|v([a, b), x)-v([a, b), y)\|_{L^{2}} \leq 4 c_{0} \Delta(x, y)
$$

for all $x, y \in S$ with $\Delta(x, y)$ small. Hence there is a constant $\eta_{0} \in\left(0, \rho_{0} / 3\right)$ such that for all $r \in\left(0, \eta_{0}\right)$ and $\varepsilon \leq r$, the minimal number of $d_{v}$-balls of radius $\varepsilon$ needed to cover $S$ is

$$
N_{\varepsilon}(S) \leq \psi(\varepsilon):=C_{N, Q}\left(\frac{r}{\varepsilon}\right)^{Q} .
$$

Note that this function $\psi(\varepsilon)$ satisfies (3.5) with the constants $c_{1}=c_{2}=2^{Q}$ which are greater than 1. It follows from Lemma 3.4 that there is a constant $K$ such that (3.7) holds. This proves Lemma 3.5.

The following is the main estimate, which is an extension of Proposition 3.4 in Talagrand [13].

Proposition 3.6. Let $c>0$ be a constant and suppose that Assumption 2.1 holds. Then there are constants $K_{1}$ and $0<\eta_{1}<1$ such that for all $0<r_{0}<\eta_{1}, \rho \in\left(0, \rho_{0} / 3\right)$, $\left(t^{1}, \ldots, t^{m}\right) \in \bar{A}_{n}$ and $\left(s^{1}, \ldots, s^{m}\right) \in B_{2 \rho}^{1} \times \cdots \times B_{2 \rho}^{m}$, we have

$$
\begin{aligned}
& \mathrm{P}\left(\exists r \in\left[r_{0}^{2}, r_{0}\right], \sup _{1 \leq i \leq m} \sup _{x^{i} \in S\left(s^{i}, c r\right)}\left|v\left(x^{i}\right)-v\left(s^{i}\right)\right| \leq K_{1} r\left(\log \log \frac{1}{r}\right)^{-1 / Q}\right) \\
& \quad \geq 1-\exp \left(-\left(\log \frac{1}{r_{0}}\right)^{1 / 2}\right) .
\end{aligned}
$$

Proof. The method of proof is similar to that of Proposition 3.4 in Talagrand [13]. For reader's convenience we provide a complete proof of Proposition 3.6 here. The main ingredients are the small ball probability estimate in Lemma 3.5 and the estimate of the approximation error in Lemma 3.2.

As in [14,13] and [5], let $U>1$ be fixed for now and its value will be chosen later. Set $r_{\ell}=r_{0} U^{-2 \ell}$ and $a_{\ell}=U^{2 \ell-1} / r_{0}$. Consider the largest integer $\ell_{0}$ such that

$$
\ell_{0} \leq \frac{\log \left(1 / r_{0}\right)}{2 \log U}
$$


Then for $\ell \leq \ell_{0}$, we have $r_{\ell} \geq r_{0}^{2}$. It suffices to show that, for some large constant $K_{1}$,

$$
\begin{aligned}
& \mathrm{P}\left(\exists 1 \leq \ell \leq \ell_{0}, \sup _{1 \leq i \leq m} \sup _{x^{i} \in S\left(s^{i}, c r_{\ell}\right)}\left|v\left(x^{i}\right)-v\left(s^{i}\right)\right| \leq K_{1} \frac{r_{\ell}}{\left(\log \log \frac{1}{r_{\ell}}\right)^{1 / Q}}\right) \\
& \quad \geq 1-\exp \left(-\left(\log \frac{1}{r_{0}}\right)^{1 / 2}\right) .
\end{aligned}
$$

It follows from Lemma 3.5 that, for $K_{1}$ large enough so that $K / K_{1}^{Q} \leq 1 / 4$,

$$
\begin{aligned}
& \mathrm{P}\left(\sup _{1 \leq i \leq m} \sup _{x^{i} \in S\left(s^{i}, c r_{\ell}\right)}\left|v\left(\left[a_{\ell}, a_{\ell+1}\right), x^{i}\right)-v\left(\left[a_{\ell}, a_{\ell+1}\right), s^{i}\right)\right| \leq K_{1} \frac{r_{\ell}}{\left(\log \log \frac{1}{r_{\ell}}\right)^{1 / Q}}\right) \\
& \geq \exp \left(-\frac{K}{K_{1}^{Q}} \log \log \frac{1}{r_{\ell}}\right) \\
& \geq\left(\log \frac{1}{r_{\ell}}\right)^{-1 / 4} .
\end{aligned}
$$

Thus, by the independence of the Gaussian processes $v\left(\left[a_{\ell}, a_{\ell+1}\right), \cdot\right)\left(\ell=1, \ldots, \ell_{0}\right)$, we have

$$
\begin{aligned}
& \mathrm{P}\left(\exists \ell \leq \ell_{0}, \sup _{1 \leq i \leq m} \sup _{x^{i} \in S\left(s^{i}, c r_{\ell}\right)}\left|v\left(\left[a_{\ell}, a_{\ell+1}\right), x^{i}\right)-v\left(\left[a_{\ell}, a_{\ell+1}\right), s^{i}\right)\right| \leq \frac{K_{1} r_{\ell}}{\left(\log \log \frac{1}{r_{\ell}}\right)^{1 / Q}}\right) \\
& =1-\prod_{\ell=1}^{\ell_{0}}\left\{1-\mathrm{P}\left(\sup _{1 \leq i \leq m} \sup _{x^{i} \in S\left(s^{i}, c r_{\ell}\right)}\left|v\left(\left[a_{\ell}, a_{\ell+1}\right), x^{i}\right)-v\left(\left[a_{\ell}, a_{\ell+1}\right), s^{i}\right)\right| \leq \frac{K_{1} r_{\ell}}{\left(\log \log \frac{1}{r_{\ell}}\right)^{1 / Q}}\right)\right\} .
\end{aligned}
$$

By (3.9), we see that the last expression is greater than or equal to

$$
\begin{aligned}
1-\prod_{\ell=1}^{\ell_{0}}\left\{1-\left(\log \frac{1}{r_{\ell}}\right)^{-1 / 4}\right\} & \geq 1-\left\{1-\left(\log \frac{1}{r_{0}^{2}}\right)^{-1 / 4}\right\}^{\ell_{0}} \\
& \geq 1-\exp \left(-\ell_{0}\left(\log \frac{1}{r_{0}^{2}}\right)^{-1 / 4}\right) .
\end{aligned}
$$

Set

$$
A_{\ell}=\sum_{j=1}^{k} a_{\ell}^{\alpha_{j}^{-1}-1} r_{\ell}^{\alpha_{j}^{-1}}+a_{\ell+1}^{-1} .
$$

Notice that $r_{\ell} a_{\ell}=U^{-1}$ and $r_{\ell} a_{\ell+1}=U$. Then

$$
A_{\ell} r_{\ell}^{-1}=\sum_{j=1}^{k}\left(a_{\ell} r_{\ell}\right)^{\alpha_{j}^{-1}-1}+\left(a_{\ell+1} r_{\ell}\right)^{-1}=\sum_{j=1}^{k} U^{-\left(\alpha_{j}^{-1}-1\right)}+U^{-1} \leq(k+1) U^{-\beta},
$$

with $\beta=\min \left\{1, \min _{j=1, \ldots, k}\left(\alpha_{j}^{-1}-1\right)\right\}>0$ since $\alpha_{j}<1$ for $j=1, \ldots, k$. Therefore, for $U$ large enough, $A_{\ell} \leq A_{0} r_{\ell}$, and for $u \geq \tilde{K} r_{\ell} U^{-\beta} \sqrt{\log U}$, (3.4) is satisfied. Hence, by Lemma 3.2 and (3.11),

$$
\begin{aligned}
& \mathrm{P}\left(\sup _{1 \leq i \leq m} \sup _{x^{i} \in S\left(s^{i}, c r \ell\right)}\left|v\left(x^{i}\right)-v\left(s^{i}\right)-v\left(\left[a_{\ell}, a_{\ell+1}\right), x^{i}\right)+v\left(\left[a_{\ell}, a_{\ell+1}\right), s^{i}\right)\right| \geq u\right) \\
& \leq \exp \left(-\frac{u^{2}}{\tilde{c} A_{\ell}^{2}}\right) \\
& \leq \exp \left(-\frac{u^{2}}{\tilde{c}(k+1)^{2} r_{\ell}^{2}} U^{2 \beta}\right) .
\end{aligned}
$$




\section{Multiple points of Gaussian random fields}

Now we take $u=K_{1} r_{\ell}\left(\log \log \frac{1}{r_{0}}\right)^{-1 / Q}$, which is allowed provided

$$
K_{1} r_{\ell}\left(\log \log \frac{1}{r_{0}}\right)^{-1 / Q} \geq \tilde{K} r_{\ell} U^{-\beta} \sqrt{\log U}
$$

This is equivalent to

$$
U^{\beta}(\log U)^{-1 / 2} \geq \frac{\tilde{K}}{K_{1}}\left(\log \log \frac{1}{r_{0}}\right)^{1 / Q},
$$

which holds if $U$ is large enough. It follows from the above that

$$
\begin{aligned}
& \mathrm{P}\left(\sup _{1 \leq i \leq m} \sup _{x^{i} \in S\left(s^{i}, c r_{\ell}\right)}\left|v\left(x^{i}\right)-v\left(s^{i}\right)-v\left(\left[a_{\ell}, a_{\ell+1}\right), x^{i}\right)+v\left(\left[a_{\ell}, a_{\ell+1}\right), s^{i}\right)\right| \geq \frac{K_{1} r_{\ell}}{\left(\log \log \frac{1}{r_{0}}\right)^{1 / Q}}\right) \\
& \quad \leq \exp \left(-\frac{U^{2 \beta}}{\tilde{c}(k+1)^{2}\left(\log \log \frac{1}{r_{0}}\right)^{2 / Q}}\right) .
\end{aligned}
$$

Let

$$
\begin{aligned}
F_{\ell} & =\left\{\sup _{1 \leq i \leq m} \sup _{x^{i} \in S\left(s^{i}, c r_{\ell}\right)}\left|v\left(\left[a_{\ell}, a_{\ell+1}\right), x^{i}\right)-v\left(\left[a_{\ell}, a_{\ell+1}\right), s^{i}\right)\right| \leq \frac{K_{1} r_{\ell}}{2\left(\log \log \frac{1}{r_{\ell}}\right)^{1 / Q}}\right\}, \\
G_{\ell} & =\left\{\sup _{1 \leq i \leq m} \sup _{x^{i} \in S\left(s^{i}, c r_{\ell}\right)}\left|v\left(x^{i}\right)-v\left(s^{i}\right)-v\left(\left[a_{\ell}, a_{\ell+1}\right), x^{i}\right)+v\left(\left[a_{\ell}, a_{\ell+1}\right), s^{i}\right)\right| \geq \frac{K_{1} r_{\ell}}{2\left(\log \log \frac{1}{r_{\ell}}\right)^{1 / Q}}\right\} .
\end{aligned}
$$

Then

$$
\begin{aligned}
& \mathrm{P}\left(\exists 1 \leq \ell \leq \ell_{0}, \sup _{1 \leq i \leq m} \sup _{x^{i} \in S\left(s^{i}, c r_{\ell}\right)}\left|v\left(x^{i}\right)-v\left(s^{i}\right)\right| \leq \frac{K_{1} r_{\ell}}{\left(\log \log \frac{1}{r_{\ell}}\right)^{1 / Q}}\right) \\
& \geq \mathrm{P}\left(\bigcup_{\ell=1}^{\ell_{0}}\left(F_{\ell} \cap G_{\ell}^{c}\right)\right) \\
& \geq \mathrm{P}\left(\left(\bigcup_{\ell=1}^{\ell_{0}} F_{\ell}\right) \cap\left(\bigcup_{\ell=1}^{\ell_{0}} G_{\ell}\right)^{c}\right) \\
& \geq \mathrm{P}\left(\bigcup_{\ell=1}^{\ell_{0}} F_{\ell}\right)-\mathrm{P}\left(\bigcup_{\ell=1}^{\ell_{0}} G_{\ell}\right) .
\end{aligned}
$$

By (3.10), we have

$$
\mathrm{P}\left(\bigcup_{\ell=1}^{\ell_{0}} F_{\ell}\right) \geq 1-\exp \left(-\ell_{0}\left(\log \frac{1}{r_{0}^{2}}\right)^{-1 / 4}\right)
$$

and by (3.13),

$$
\mathrm{P}\left(\bigcup_{\ell=1}^{\ell_{0}} G_{\ell}\right) \leq \ell_{0} \exp \left(-\frac{U^{2 \beta}}{\tilde{c}(k+1)^{2}\left(\log \log \frac{1}{r_{0}}\right)^{2 / Q}}\right) .
$$

Combining this with (3.14), we get

$$
\begin{aligned}
& \mathrm{P}\left(\exists 1 \leq \ell \leq \ell_{0}, \sup _{1 \leq i \leq m} \sup _{x^{i} \in S\left(s^{i}, c r_{\ell}\right)}\left|v\left(x^{i}\right)-v\left(s^{i}\right)\right| \leq \frac{K_{1} r_{\ell}}{\left(\log \log \frac{1}{r_{\ell}}\right)^{1 / Q}}\right) \\
& \geq 1-\exp \left(-\ell_{0}\left(\log \frac{1}{r_{0}^{2}}\right)^{-1 / 4}\right)-\ell_{0} \exp \left(-\frac{U^{2 \beta}}{\tilde{c}(k+1)^{2}\left(\log \log \frac{1}{r_{0}}\right)^{2 / Q}}\right) .
\end{aligned}
$$


Therefore, the proof will be completed provided

$$
\begin{aligned}
& \exp \left(-\ell_{0}\left(\log \frac{1}{r_{0}^{2}}\right)^{-1 / 4}\right)+\ell_{0} \exp \left(-\frac{U^{2 \beta}}{\tilde{c}(k+1)^{2}\left(\log \log \frac{1}{r_{0}}\right)^{2 / Q}}\right) \\
& \leq \exp \left(-\left(\log \frac{1}{r_{0}}\right)^{1 / 2}\right) .
\end{aligned}
$$

Recall the condition (3.12), and the definition of $\ell_{0}$ in (3.8). If we set

$$
U=\left(\log \frac{1}{r_{0}}\right)^{1 /(2 \beta)},
$$

then for $r_{0}$ small enough, by (3.8),

$$
\ell_{0}>\frac{\beta}{2}\left(\log \frac{1}{r_{0}}\right)\left(\log \log \frac{1}{r_{0}}\right)^{-1}>1 .
$$

Therefore, the left-hand side of (3.15) is bounded above by

$$
\begin{aligned}
& \exp \left(-\frac{\left(\log \frac{1}{r_{0}}\right)^{3 / 4}}{\tilde{c}(k+1)^{2} \log \log \frac{1}{r_{0}}}\right)+\left(1+\log \frac{1}{r_{0}}\right) \exp \left(-\frac{\log \frac{1}{r_{0}}}{\tilde{c}(k+1)^{2}\left(\log \log \frac{1}{r_{0}}\right)^{2 / Q}}\right) \\
& \leq \exp \left(-\left(\log \frac{1}{r_{0}}\right)^{1 / 2}\right)
\end{aligned}
$$

provided $r_{0}$ is small enough. This completes the proof of Proposition 3.6.

Let $n \geq 1$ be fixed. Notice that if $\left(t^{1}, \ldots, t^{m}\right) \in A_{n}$ and $0<\rho<\frac{1}{2 c^{\prime} n}$, where $c^{\prime}>0$ is the constant in Assumption 2.2, then $\Delta\left(t^{i}, t^{h}\right) \geq 2 c^{\prime} \rho$ for $i \neq h$. It follows from Assumption 2.2 that for each $\left(t^{1}, \ldots, t^{m}\right) \in A_{n}$ and $\rho \in\left(0, \varepsilon_{0} \wedge \frac{1}{2 c^{\prime} n}\right)$, there are $\left(\hat{t}^{1}, \ldots, \hat{t}^{m}\right) \in B_{c \rho}^{1} \times \cdots \times B_{c \rho}^{m}$ (recall that $B_{\rho}^{i}=B_{\rho}\left(t^{i}\right)$ ) such that for all $h=1, \ldots, m$ and all $x, y \in B_{2 \rho}^{i}(i=1, \ldots, m)$, we have

$$
\left|\mathrm{E}\left((v(x)-v(y)) \cdot v\left(\hat{t}^{h}\right)\right)\right| \leq C \sum_{j=1}^{k}\left|x_{j}-y_{j}\right|^{\delta_{j}} .
$$

The points $\hat{t}^{1}, \ldots, \hat{t}^{m}$ are determined by $t^{1}, \ldots, t^{m}$, and $\left(\hat{t}^{1}, \ldots, \hat{t}^{m}\right) \in \bar{A}_{2 n}$ provided $0<$ $\rho<\varepsilon_{0} \wedge \frac{1}{2 c^{\prime} n}$, where $a \wedge b=\min \{a, b\}$.

Let $\Sigma_{2}$ denote the $\sigma$-algebra generated by $v\left(\hat{t}^{1}\right), \ldots, v\left(\hat{t}^{m}\right)$. Define

$$
v^{2}(x)=\mathrm{E}\left(v(x) \mid \Sigma_{2}\right), \quad v^{1}(x)=v(x)-v^{2}(x) .
$$

The Gaussian random fields $v^{1}=\left\{v^{1}(x), x \in T\right\}$ and $v^{2}=\left\{v^{2}(x), x \in T\right\}$ are independent.

Lemma 3.7. Suppose Assumptions 2.1, 2.2 and 2.3 are satisfied. For any $0<\rho<$ $\varepsilon_{0} \wedge \frac{1}{2 c^{\prime} n}$, there is a constant $K_{2}$ depending on $n$ and the constants $C$ in Assumption 2.2 (but not on $\hat{t}^{1}, \ldots, \hat{t}^{m}$ ) such that for all $i=1, \ldots, m$ and all $x, y \in B_{2 \rho}^{i}$,

$$
\left|v^{2}(x)-v^{2}(y)\right| \leq K_{2} \sum_{j=1}^{k}\left|x_{j}-y_{j}\right|^{\delta_{j}} \max _{1 \leq \ell \leq m}\left|v\left(\hat{t}^{\ell}\right)\right| .
$$

Proof. By Assumption 2.3, the subspace in $L^{2}\left(\Omega ; \mathbb{R}^{d}\right)$ of random vectors $\Omega \rightarrow \mathbb{R}^{d}$ spanned by $v\left(\hat{t}^{1}\right), \ldots, v\left(\hat{t}^{m}\right)$, has dimension $m \geq 2$. Let $\left\{\sum_{h=1}^{m} a_{h, j} v\left(\hat{t}^{h}\right): j=1, \ldots, m\right\}$ be an orthonormal basis of this subspace obtained by the Gram-Schmidt orthogonalization 
procedure, so that the coefficients $a_{i, j}$ are continuous functions of $\left(\hat{t}^{1}, \ldots, \hat{t}^{m}\right) \in \bar{A}_{2 n}$. Then

$$
v^{2}(x)=\sum_{j=1}^{m} \mathrm{E}\left[\sum_{h=1}^{m} a_{h, j} v\left(\hat{t}^{h}\right) \cdot v(x)\right]\left(\sum_{\ell=1}^{m} a_{\ell, j} v\left(\hat{t}^{\ell}\right)\right) .
$$

By (3.16), for all $i=1, \ldots, m$ and all $x, y \in B_{2 \rho}^{i}$,

$$
\begin{aligned}
\left|v^{2}(x)-v^{2}(y)\right| & =\left|\sum_{\ell=1}^{m}\left(\sum_{h=1}^{m} \sum_{j=1}^{m} a_{h, j} a_{\ell, j} \mathrm{E}\left[(v(x)-v(y)) \cdot v\left(\hat{t}^{h}\right)\right]\right) v\left(\hat{t}^{\ell}\right)\right| \\
& \leq K \sum_{j=1}^{k}\left|x_{j}-y_{j}\right|^{\delta_{j}} \max _{1 \leq \ell \leq m}\left|v\left(\hat{t}^{\ell}\right)\right| .
\end{aligned}
$$

By the continuity of $a_{i, j}$ in $\left(\hat{t}^{1}, \ldots, \hat{t}^{m}\right)$ and the compactness of $\bar{A}_{2 n}$, we see that the constant $K$ is independent of $\hat{t}^{1}, \ldots, \hat{t}^{m}$. This completes the proof.

Lemma 3.8. Suppose Assumptions 2.1, 2.2 and 2.3 are satisfied. Let $n \geq 1$. Then there exist constants $K$ and $\rho_{0} \in(0,1 / n)$ which may depend on $n$ such that for all $\rho \in\left(0, \rho_{0}\right)$, $a_{2}, \ldots, a_{m} \in \mathbb{R}^{d}, r>0,\left(t^{1}, \ldots, t^{m}\right) \in \bar{A}_{n}$ and all $\left(x^{1}, \ldots, x^{m}\right) \in B_{\rho}\left(t^{1}\right) \times \cdots \times B_{\rho}\left(t^{m}\right)$,

$$
\mathrm{P}\left(\sup _{2 \leq i \leq m}\left|v^{2}\left(x^{1}\right)-v^{2}\left(x^{i}\right)-a_{i}\right| \leq r\right) \leq K r^{(m-1) d} .
$$

Proof. We first assume $d=1$. We claim that if $\rho_{0}$ is small then $v^{2}\left(x^{1}\right), \ldots, v^{2}\left(x^{m}\right)$ are linearly independent for all $\rho \in\left(0, \rho_{0}\right),\left(t^{1}, \ldots, t^{m}\right) \in \bar{A}_{n}$ and $\left(x^{1}, \ldots, x^{m}\right) \in B_{\rho}\left(t^{1}\right) \times$ $\cdots \times B_{\rho}\left(t^{m}\right)$. Indeed, by Assumption 2.3 and the compactness of $\bar{A}_{n}$, we can find $C>0$ depending on $n$ such that $\operatorname{Var}\left(\sum_{i=1}^{m} b_{i} v\left(t^{i}\right)\right) \geq C$ for all $\left(t^{1}, \ldots, t^{m}\right) \in \bar{A}_{n}$ and $b \in \mathbb{R}^{m}$ with $|b|=1$. Then by the Cauchy-Schwarz inequality, we have

$$
\begin{aligned}
& {\left[\mathrm{E}\left(\sum_{i=1}^{m} b_{i}\left(v\left(t^{i}\right)-v^{2}\left(x^{i}\right)\right)\right)^{2}\right]^{1 / 2} \leq|b|\left[\mathrm{E}\left(\sum_{i=1}^{m}\left(v\left(t^{i}\right)-v^{2}\left(x^{i}\right)\right)^{2}\right)\right]^{1 / 2}} \\
& \quad \leq|b| \sum_{i=1}^{m}\left(\left[\mathrm{E}\left(v\left(t^{i}\right)-v\left(\hat{t}^{i}\right)\right)^{2}\right]^{1 / 2}+\left[\mathrm{E}\left(\mathrm{E}\left(v\left(\hat{t}^{i}\right)-v\left(x^{i}\right) \mid \Sigma_{2}\right)\right)^{2}\right]^{1 / 2}\right) \\
& \quad \leq|b| \sum_{i=1}^{m}\left(\left\|v\left(t^{i}\right)-v\left(\hat{t}^{i}\right)\right\|_{L^{2}}+\left\|v\left(\hat{t}^{i}\right)-v\left(x^{i}\right)\right\|_{L^{2}}\right) .
\end{aligned}
$$

It follows that

$$
\begin{aligned}
{\left[\mathrm{E}\left(\sum_{i=1}^{m} b_{i} v^{2}\left(x^{i}\right)\right)\right]^{1 / 2} } & \geq\left[\mathrm{E}\left(\sum_{i=1}^{m} b_{i} v\left(t^{i}\right)\right)^{2}\right]^{1 / 2}-\left[\mathrm{E}\left(\sum_{i=1}^{m} b_{i}\left(v\left(t^{i}\right)-v^{2}\left(x^{i}\right)\right)\right)^{2}\right]^{1 / 2} \\
& \geq\left(C^{1 / 2}-\sum_{i=1}^{m}\left(\left\|v\left(t^{i}\right)-v\left(\hat{t}^{i}\right)\right\|_{L^{2}}+\left\|v\left(\hat{t}^{i}\right)-v\left(x^{i}\right)\right\|_{L^{2}}\right)\right)|b| .
\end{aligned}
$$

Notice that, Assumption 2.1 implies the $L^{2}(\mathrm{P})$-continuity of $v(x)$ [cf. Lemma 3.1], we can find a small constant $\rho_{0} \in(0,1 / n)$ depending on $C$ so that the above is $\geq C^{\prime}|b|$ for all $\rho \in\left(0, \rho_{0}\right),\left(t^{1}, \ldots, t^{m}\right) \in \bar{A}_{n}$ and $\left(x^{1}, \ldots, x^{m}\right) \in B_{\rho}\left(t^{1}\right) \times \cdots \times B_{\rho}\left(t^{m}\right)$, where $C^{\prime}>0$. It follows that $v^{2}\left(x^{1}\right), \ldots, v^{2}\left(x^{m}\right)$ are linearly independent, and so are $v^{2}\left(x^{1}\right)-$ $v^{2}\left(x^{2}\right), v^{2}\left(x^{1}\right)-v^{2}\left(x^{3}\right), \ldots, v^{2}\left(x^{1}\right)-v^{2}\left(x^{m}\right)$.

Denote the determinant of the covariance matrix of the last random vector by

$$
\operatorname{det} \operatorname{Cov}\left(v^{2}\left(x^{1}\right)-v^{2}\left(x^{2}\right), v^{2}\left(x^{1}\right)-v^{2}\left(x^{3}\right), \ldots, v^{2}\left(x^{1}\right)-v^{2}\left(x^{m}\right)\right) .
$$


If $\rho \in\left(0, \rho_{0}\right),\left(t^{1}, \ldots, t^{m}\right) \in \bar{A}_{n}$ and $\left(x^{1}, \ldots, x^{m}\right) \in B_{\rho}\left(t^{1}\right) \times \cdots \times B_{\rho}\left(t^{m}\right)$, then $\left(x^{1}, \ldots, x^{m}\right) \in$ $\bar{A}_{2 n}$ provided that $\rho_{0}$ is small. Since the function

$$
\left(x^{1}, \ldots, x^{m}\right) \mapsto \operatorname{det} \operatorname{Cov}\left(v^{2}\left(x^{1}\right)-v^{2}\left(x^{2}\right), v^{2}\left(x^{1}\right)-v^{2}\left(x^{3}\right), \ldots, v^{2}\left(x^{1}\right)-v^{2}\left(x^{m}\right)\right)
$$

is continuous and positive on the compact set $\bar{A}_{2 n}$, it is bounded from below by a positive constant depending on $n$. This and Anderson's theorem [1] imply that

$$
\mathrm{P}\left(\sup _{2 \leq i \leq m}\left|v^{2}\left(x^{1}\right)-v^{2}\left(x^{i}\right)-a_{i}\right| \leq r\right) \leq \mathrm{P}\left(\sup _{2 \leq i \leq m}\left|v^{2}\left(x^{1}\right)-v^{2}\left(x^{i}\right)\right| \leq r\right) \leq K r^{m-1} .
$$

Since $v(x)$ has i.i.d. components, the case $d>1$ follows readily.

We end this section with the following lemma which is obtained by applying Theorem 2.1 and Remark 2.2 of [7] to the metric space $(T, \Delta)$. It provides nested families of "cubes" sharing most of the good properties of dyadic cubes in the Euclidean spaces. For this reason, we call the sets in $\mathscr{Q}_{q}$ generalized dyadic cubes of order $q$. Their nesting property will help us to construct an economic covering for $M_{\rho}$.

Lemma 3.9. There exist constants $c_{1}, c_{2}$, and a family $\mathscr{Q}$ of Borel subsets of $T$, where $\mathscr{Q}=\bigcup_{q=1}^{\infty} \mathscr{Q}_{q}, \mathscr{Q}_{q}=\left\{I_{q, \ell}: \ell=1, \ldots, n_{q}\right\}$, such that the following hold.

(i) $T=\bigcup_{\ell=1}^{n_{q}} I_{q, \ell}$ for each $q \geq 1$.

(ii) Either $I_{q, \ell} \cap I_{q^{\prime}, \ell^{\prime}}=\varnothing$ or $I_{q, \ell} \subset I_{q^{\prime}, \ell^{\prime}}$ whenever $q \geq q^{\prime}, 1 \leq \ell \leq n_{q}, 1 \leq \ell^{\prime} \leq n_{q^{\prime}}$.

(iii) For each $q, \ell$, there exists $x_{q, \ell} \in T$ such that $S\left(x_{q, \ell}, c_{1} 2^{-q}\right) \subset I_{q, \ell} \subset S\left(x_{q, \ell}, c_{2} 2^{-q}\right)$ and $\left\{x_{q, \ell}: 1, \ldots, n_{q}\right\} \subset\left\{x_{q+1, \ell}: \ell=1, \ldots, n_{q+1}\right\}$ for all $q \geq 1$.

\section{Proof of Theorem 2.5}

Recall that, by (3.3), it suffices to show that for all integers $n$, we can find a small $\rho_{0}>0$ such that for all $\rho \in\left(0, \rho_{0}\right)$ and all points $\left(t^{1}, \ldots, t^{m}\right) \in A_{n}, M_{\rho}$ is empty with probability 1 . When $m Q<(m-1) d$ (we refer to this as the sub-critical case), the last statement can be proved easily by using a standard covering argument based on the uniform modulus of continuity of $v=\{v(x), x \in T\}$ on compact intervals. In the following we provide a unified proof for both the critical and sub-critical cases.

For any $n \geq 1$ fixed, we choose a constant $\rho_{0}>0$ such that Assumption 2.2, Lemma 3.7 and Lemma 3.8 hold for all $\rho \leq \rho_{0}$ (e.g., we take $\left.\rho_{0} \leq \varepsilon_{0} \wedge \frac{1}{2 c^{\prime} n}\right)$. Let $\left(t^{1}, \ldots, t^{m}\right) \in A_{n}$ be fixed in the rest of the proof. By Assumption 2.2, we can find $\left(\hat{t}^{1}, \ldots, \hat{t}^{m}\right) \in B_{c \rho}^{1} \times \cdots \times B_{c \rho}^{m}$ such that (3.16) holds. Furthermore, we assume that $B_{c \rho_{0}}^{j} \subset T$ for all $1 \leq j \leq m$ (otherwise we take the intersection with $T$ ).

Fix $\rho \in\left(0, \rho_{0}\right)$. For each integer $p \geq 1$, consider the random set

$$
\begin{aligned}
R_{p}=\left\{\left(s^{1}, \ldots, s^{m}\right) \in B_{2 \rho}^{1} \times \cdots \times B_{2 \rho}^{m}: \exists r \in\left[2^{-2 p}, 2^{-p}\right]\right. \text { such that } \\
\left.\sup _{1 \leq i \leq m} \sup _{x^{i} \in S\left(s^{i}, 4 c_{2} r\right)}\left|v\left(x^{i}\right)-v\left(s^{i}\right)\right| \leq K_{1} r\left(\log \log \frac{1}{r}\right)^{-1 / Q}\right\},
\end{aligned}
$$

where $c_{2}$ is the constant given by Lemma 3.9. Let $\lambda$ denote the Lebesgue measure on $\mathbb{R}^{m k}$. Consider the event

$$
\Omega_{p, 1}=\left\{\lambda\left(R_{p}\right) \geq \lambda\left(B_{2 \rho}^{1} \times \cdots \times B_{2 \rho}^{m}\right)(1-\exp (-\sqrt{p} / 4))\right\} .
$$


This event states that a very large portion of $B_{2 \rho}^{1} \times \cdots \times B_{2 \rho}^{m}$ is taken by the random set $R_{p}$, which is the collection of points at which the sample function $v(x)$ has small oscillations. The points in $R_{p}$ are referred to as "good points" for $v$. By Markov's inequality,

$$
\begin{aligned}
\mathrm{P}\left(\Omega_{p, 1}^{c}\right) & =\mathrm{P}\left\{\lambda\left(B_{2 \rho}^{1} \times \cdots \times B_{2 \rho}^{m} \backslash R_{p}\right)>\lambda\left(B_{2 \rho}^{1} \times \cdots \times B_{2 \rho}^{m}\right) \exp (-\sqrt{p} / 4)\right\} \\
& \leq \frac{\mathrm{E}\left[\lambda\left(B_{2 \rho}^{1} \times \cdots \times B_{2 \rho}^{m} \backslash R_{p}\right)\right]}{\lambda\left(B_{2 \rho}^{1} \times \cdots \times B_{2 \rho}^{m}\right) \exp (-\sqrt{p} / 4)} .
\end{aligned}
$$

Then by Fubini's theorem, the numerator is equal to

$$
\mathrm{E}\left[\lambda\left(B_{2 \rho}^{1} \times \cdots \times B_{2 \rho}^{m} \backslash R_{p}\right)\right]=\int_{B_{2 \rho}^{1} \times \cdots \times B_{2 \rho}^{m}} \mathrm{P}\left(\left(s^{1}, \ldots, s^{m}\right) \in B_{2 \rho}^{1} \times \cdots \times B_{2 \rho}^{m} \backslash R_{p}\right) d s^{1} \cdots d s^{m} .
$$

By applying Proposition 3.6 with $c=4 c_{2}$, we derive that for $p$ sufficiently large,

$$
\mathrm{P}\left(\left(s^{1}, \ldots, s^{m}\right) \in R_{p}\right) \geq 1-\exp (-\sqrt{p} / 2)
$$

for all $\left(s^{1}, \ldots, s^{m}\right) \in B_{2 \rho}^{1} \times \cdots \times B_{2 \rho}^{m}$. Hence $\mathrm{P}\left(\Omega_{p, 1}^{c}\right) \leq \exp (-\sqrt{p} / 4)$ and $\sum_{p=1}^{\infty} \mathrm{P}\left(\Omega_{p, 1}^{c}\right)<\infty$.

Fix $\beta \in\left(0, \beta^{*} \wedge 1\right)$, where $\beta^{*}=\min _{1 \leq j \leq k}\left\{\frac{\delta_{j}}{\alpha_{j}}-1\right\}$. Consider the event

$$
\Omega_{p, 2}=\left\{\max _{1 \leq i \leq m}\left|v\left(\hat{t}^{i}\right)\right| \leq 2^{\beta p}\right\} .
$$

By Lemma 3.7, we can control the oscillation of $v^{2}(x)$ on $\Omega_{p, 2}$. It is clear that $\sum_{p=1}^{\infty} \mathrm{P}\left(\Omega_{p, 2}^{c}\right)$ $<\infty$.

For the points which are not in the random set $R_{p}$, the sample function $v(x)$ may have large oscillations in their neighborhoods. These points are referred to as "bad points" for $v$. In order to quantify their effect we introduce the following event $\Omega_{p, 3}$ :

$$
\Omega_{p, 3}=\left\{\forall I \in \mathscr{Q}_{2 p}, \sup _{x, y \in I}|v(x)-v(y)| \leq K_{3} 2^{-2 p} p^{1 / 2}\right\} .
$$

Recall that $\mathscr{Q}=\bigcup_{q=1}^{\infty} \mathscr{Q}_{q}$ is the family of generalized dyadic cubes given by Lemma 3.9 for the compact interval $T$.

For every $I \in \mathscr{Q}_{2 p}$, Lemma 3.1 implies that the diameter of $I$ under the canonical metric $d_{v}(x, y)=\|v(x)-v(y)\|_{L^{2}}$ is at most $c_{3} 2^{-2 p}$. By applying Lemma 2.1 in Talagrand [14] (see also Lemma 3.1 in [5]) we see that for any positive constant $K_{3}$ and $p$ large,

$$
\mathrm{P}\left(\sup _{x, y \in I}|v(x)-v(y)| \geq K_{3} 2^{-2 p} p^{1 / 2}\right) \leq \exp \left(-\left(\frac{K_{3}}{c_{3}}\right)^{2} p\right) .
$$

Notice that the cardinality of the family $\mathscr{Q}_{2 p}$ of generalized dyadic cubes of order $2 p$ is at most $K 2^{2 p Q}$. We can verify directly that $\sum_{p=1}^{\infty} \mathrm{P}\left(\Omega_{p, 3}^{c}\right)<\infty$ provided $K_{3}$ is chosen to satisfy $K_{3}>2 c_{3} Q \ln 2$.

Let $\Omega_{p}=\Omega_{p, 1} \cap \Omega_{p, 2} \cap \Omega_{p, 3}$ and

$$
\Omega^{*}=\bigcup_{\ell \geq 1} \bigcap_{p \geq \ell} \Omega_{p}
$$

It follows from the above that the event $\Omega^{*}$ occurs with probability 1 . Hence, almost surely $\Omega_{p}$ occurs for all $p$ large enough. Notice that on the event $\Omega_{p}$, the oscillations of $v$ near the good and bad points can be explicitly controlled by the inequalities in (4.1) and (4.2), respectively. Moreover, because of Lemma 3.7 and $\Omega_{p, 2}$, it can be verified that 
similar inequalities (with constants larger than $K_{1}$ and $K_{3}$ ) hold for $v^{1}$. In the following, we will use these observations to show that, for every $\omega \in \Omega^{*}$, we can construct families of balls in $\mathbb{R}^{d}$ that cover $M_{\rho}$.

For each $p \geq 1$, we first construct a family $\mathscr{G}_{p}$ of subsets in $\mathbb{R}^{m k}$ (depending on $\omega$ ). Denote by $\mathscr{C}_{p}$ the family of subsets of $T^{m}$ of the form $C=I_{q, \ell_{1}} \times \cdots \times I_{q, \ell_{m}}$ for some integer $q \in[p, 2 p]$, where $I_{q, \ell_{i}} \in \mathscr{Q}_{q}$ are the generalized dyadic cubes of order $q$ in Lemma 3.9 .

We say that a dyadic cube $C=I^{1} \times \cdots \times I^{m}$ of order $q$ is good if it has the property that

$$
\sup _{1 \leq i \leq m} \sup _{x, y \in I^{i}}\left|v^{1}(x)-v^{1}(y)\right| \leq d_{q}
$$

where

$$
d_{q}=2\left(K_{1}+K_{2} \sum_{j=1}^{k}\left(2 c_{2}\right)^{\delta_{j} / \alpha_{j}}\right) 2^{-q}\left(\log \log 2^{q}\right)^{-1 / Q} .
$$

For each $x \in B_{2 \rho}^{1} \times \cdots \times B_{2 \rho}^{m}$, consider the good dyadic cube $C$ containing $x$ (if any) of smallest order $q$, where $p \leq q \leq 2 p$. By property (ii) of Lemma 3.9, we obtain in this way a family of disjoint good dyadic cubes of order $q \in[p, 2 p]$ that meet the set $B_{2 \rho}^{1} \times \cdots \times B_{2 \rho}^{m}$. We denote this family by $\mathscr{G}_{p}^{1}$.

Let $\mathscr{G}_{p}^{2}$ be the family of dyadic cubes in $T^{m}$ of order $2 p$ that meet $B_{\rho}^{1} \times \cdots \times B_{\rho}^{m}$ but are not contained in any cube of $\mathscr{G}_{p}^{1}$. Let $\mathscr{G}_{p}=\mathscr{G}_{p}^{1} \cup \mathscr{G}_{p}^{2}$. Notice that for each $C \in \mathscr{C}_{p}$, the events $\left\{C \in \mathscr{G}_{p}^{1}\right\}$ and $\left\{C \in \mathscr{G}_{p}^{2}\right\}$ are in the $\sigma$-algebra $\Sigma_{1}:=\sigma\left\{v^{1}(x): x \in T\right\}$.

Next we construct a family of balls in $\mathbb{R}^{d}$ (depending on $\omega$ ) as follows. For each $C \in \mathscr{C}_{p}$, we choose a distinguished (non-random) point $x_{C}=\left(x_{C}^{1}, \ldots, x_{C}^{m}\right)$ in $C \cap\left(B_{2 \rho}^{1} \times \cdots \times B_{2 \rho}^{m}\right)$. If $C$ is a cube of order $q$, then we define the ball $B_{p, C}$ as follows.

(i) If $C \in \mathscr{G}_{p}^{1}$, take $B_{p, C}$ as the Euclidean ball in $\mathbb{R}^{d}$ of center $v\left(x_{C}^{1}\right)$ of radius $r_{p, C}=4 d_{q}$. Recall that $d_{q}$ is defined in (4.4).

(ii) If $C \in \mathscr{G}_{p}^{2}$, take $B_{p, C}$ as the Euclidean ball in $\mathbb{R}^{d}$ of center $v\left(x_{C}^{1}\right)$ of radius $r_{p, C}=$ $2 K_{3} 2^{-2 p} p^{1 / 2}$

(iii) Otherwise, take $B_{p, C}=\varnothing$ and $r_{p, C}=0$.

Note that for each $p \geq 1$ and $C \in \mathscr{C}_{p}$, the random variable $r_{p, C}$ is $\Sigma_{1}$-measurable.

Intuitively, if a ball $B_{p, C}$ in $\mathbb{R}^{d}$ defined above contains a multiple point of $v$ from $C \cap\left(B_{2 \rho}^{1} \times \cdots \times B_{2 \rho}^{m}\right)$ (i.e., there exists $\left(y^{1}, \ldots, y^{m}\right) \in C \cap\left(B_{2 \rho}^{1} \times \cdots \times B_{2 \rho}^{m}\right)$ such that $\left.v\left(y^{1}\right)=\cdots=v\left(y^{m}\right) \in B_{p, C}\right)$, then $v\left(x_{C}^{1}\right), v\left(x_{C}^{2}\right), \ldots, v\left(x_{C}^{m}\right)$ should be close to each other because $\left|v\left(x^{i}\right)-v\left(y^{i}\right)\right|$ is small for $1 \leq i \leq k$. Hence, in order to construct a covering $\mathscr{F}_{p}(\omega)$ of the set $M_{\rho}$ of multiple points, we consider the event

$$
\Omega_{p, C}=\left\{\omega \in \Omega: \sup _{2 \leq i \leq m}\left|v\left(x_{C}^{1}, \omega\right)-v\left(x_{C}^{i}, \omega\right)\right| \leq r_{p, C}(\omega)\right\} .
$$

This is the event that $v\left(x_{C}^{2}\right), \ldots, v\left(x_{C}^{m}\right)$ are all within the ball of radius $r_{p, C}$ centered at $v\left(x_{C}^{1}\right)$.

If $\omega \in \Omega_{p} \cap \Omega_{p, C}$, define $\mathscr{F}_{p}(\omega)=\left\{B_{p, C}: C \in \mathscr{G}_{p}(\omega)\right\}$. Otherwise, define $\mathscr{F}_{p}(\omega)=\varnothing$.

Choose an integer $p_{0}$ such that

$$
2 c_{2} 2^{-p} \leq \rho \text { and } \exp (-\sqrt{p} / 4) p^{m Q}(\log p)^{m} \leq \rho^{m Q},
$$

and $\Omega_{p}$ occurs for all $p \geq p_{0}$. We now show that $\mathscr{F}_{p}(\omega)$ covers $M_{\rho}(\omega)$ whenever $p \geq p_{0}$ and $\omega \in \Omega_{p}$.

Let $\omega \in \Omega_{p}$ and $z \in M_{\rho}(\omega)$. By definition, we can find a point $\left(y^{1}, \ldots, y^{m}\right) \in B_{\rho}^{1} \times \cdots \times$ $B_{\rho}^{m}$ such that $z=v\left(y^{1}, \omega\right)=\cdots=v\left(y^{m}, \omega\right)$. By the definitions of $\mathscr{G}_{p}^{1}$ and $\mathscr{G}_{p}^{2}$, the family 
$\mathscr{G}_{p}(\omega)$ of dyadic cubes covers $B_{\rho}^{1} \times \cdots \times B_{\rho}^{m}$, thus the point $\left(y^{1}, \ldots, y^{m}\right)$ is contained in some $C=I^{1} \times \cdots \times I^{m} \in \mathscr{G}_{p}(\omega)$. We will show that $z \in B_{p, C}$ and $\omega \in \Omega_{p, C}$. To this end, we distinguish two cases.

Case 1. If $C \in \mathscr{G}_{p}^{1}(\omega)$, then it is a good dyadic cube of order $q \in[p, 2 p]$ such that

$$
\sup _{1 \leq i \leq m}\left|v^{1}\left(x_{C}^{i}, \omega\right)-v^{1}\left(y^{i}, \omega\right)\right| \leq d_{q}
$$

By Lemma 3.9, $x_{C}^{i}, y^{i} \in I^{i} \subset S\left(x^{*}, c_{2} 2^{-q}\right)$ for some $x^{*} \in T$, so we have

$$
\sum_{j=1}^{k}\left|x_{C, j}^{i}-y_{j}^{i}\right|^{\delta_{j}} \leq \sum_{j=1}^{k}\left(2 c_{2}\right)^{\delta_{j} / \alpha_{j}} 2^{-q\left(1+\beta^{*}\right)}
$$

recall that $\beta^{*}=\min _{1 \leq j \leq k}\left\{\frac{\delta_{j}}{\alpha_{j}}-1\right\}$. Since $\omega \in \Omega_{p, 2}$, Lemma 3.7 and (4.6) imply that

$$
\sup _{1 \leq i \leq m}\left|v^{2}\left(x_{C}^{i}\right)-v^{2}\left(y^{i}\right)\right| \leq K_{2} \sum_{j=1}^{k}\left(2 c_{2}\right)^{\delta_{j} / \alpha_{j}} 2^{-q\left(1+\beta^{*}-\beta\right)} \leq d_{q}
$$

since $\beta<\beta^{*}$. It follows that

$$
\sup _{1 \leq i \leq m}\left|v\left(x_{C}^{i}, \omega\right)-z\right|=\sup _{1 \leq i \leq m}\left|v\left(x_{C}^{i}, \omega\right)-v\left(y^{i}, \omega\right)\right| \leq 2 d_{q},
$$

which implies that $z \in B_{p, C}$ and $\omega \in \Omega_{p, C}$.

Case 2. Now we assume $C \in \mathscr{G}_{p}^{2}(\omega)$. Since $\omega \in \Omega_{p, 3}$, we have

$$
\sup _{i}\left|v\left(x_{C}^{i}, \omega\right)-z\right|=\sup _{i}\left|v\left(x_{C}^{i}, \omega\right)-v\left(y^{i}, \omega\right)\right| \leq K_{3} 2^{-2 p} p^{1 / 2},
$$

hence $z \in B_{p, C}$ and $\omega \in \Omega_{p, C}$.

Therefore, for every $\omega \in \Omega^{*}, \mathscr{F}_{p}(\omega)$ covers $M_{\rho}(\omega)$ for all $p$ large enough. We claim that, with probability 1 , the family $\mathscr{F}_{p}$ is empty for infinitely many $p$. This will imply that $M_{\rho}$ is empty with probability 1 and the proof will then be complete.

We prove the aforementioned claim by contradiction. Suppose the claim is not true. Then the event $\Omega^{\prime}$ that $\mathscr{F}_{p}$ is nonempty for all large $p$ has positive probability and the event $\Omega^{\prime} \cap \Omega^{*}=\bigcup_{\ell \geq 1} \bigcap_{p \geq \ell}\left(\Omega^{\prime} \cap \Omega_{p}\right)$ also has positive probability. Denote

$$
\phi(r)=r^{m Q-(m-1) d}(\log \log (1 / r))^{m}, \quad f(r)=r^{m Q}(\log \log (1 / r))^{m},
$$

and consider the random variables $X_{p}$ defined by

$$
X_{p}:=\mathbf{1}_{\Omega^{\prime} \cap \Omega_{p}} \sum_{B_{p, C} \in \mathscr{F}_{p}} \phi\left(r_{p, C}\right)=\mathbf{1}_{\Omega^{\prime} \cap \Omega_{p}} \sum_{C \in \mathscr{C}_{p}} f\left(r_{p, C}\right) r_{p, C}^{-(m-1) d} \mathbf{1}_{\left\{C \in \mathscr{G}_{p}\right\}} \mathbf{1}_{\Omega_{p, C}}
$$

Let $X:=\liminf _{p} X_{p}$. Since $m Q \leq(m-1) d$, we have $\phi(r) \rightarrow \infty$ as $r \rightarrow 0+$. Also, for every $\omega \in \Omega^{\prime} \cap \Omega^{*}, \mathscr{F}_{p}(\omega)$ is not empty for all large $p$. Hence, by the definition of $X_{p}$ in (4.8), $X(\omega)=\infty$ on $\Omega^{\prime} \cap \Omega^{*}$. In particular, $\mathrm{E}(X)=\infty$.

On the other hand, notice that $\mathscr{G}_{p}^{1}$ covers $R_{p}$ on the event $\Omega_{p}$ for all $p \geq p_{0}$. Indeed, if $\omega \in \Omega_{p}, s=\left(s^{1}, \ldots, s^{m}\right) \in R_{p}(\omega)$, and $C=I^{1} \times \cdots \times I^{m}$ is the dyadic cube of order $q$ in $\mathscr{G}_{p}^{1}$ containing $s$, then there exists $r \in\left[2^{-2 p}, 2^{-p}\right]$ that satisfies the condition in the definition of $R_{p}$ and we can find $q$ such that $2^{-q-1}<r \leq 2^{-q}, p \leq q \leq 2 p$, and

$$
\sup _{1 \leq i \leq m} \sup _{x^{i} \in S\left(s^{i}, 2 c_{2} 2^{-q}\right)}\left|v\left(x^{i}\right)-v\left(s^{i}\right)\right| \leq K_{1} 2^{-q}\left(\log \log 2^{q}\right)^{-1 / Q} .
$$


By the property that $I^{i} \subset S\left(x^{\prime}, c_{2} 2^{-q}\right)$ for some $x^{\prime}$ and by Lemma 3.7, it follows from (4.7) and (4.9) that (4.3) holds. Thus $C$ is a good dyadic cube. This proves that $\mathscr{G}_{p}^{1}(\omega)$ covers $R_{p}(\omega)$.

By the choice of $p_{0}$ in (4.5), the cubes in $\mathscr{G}_{p}^{2}$ are contained in $B_{2 \rho}^{1} \times \cdots \times B_{2 \rho}^{m}$, and thus in $B_{2 \rho}^{1} \times \cdots \times B_{2 \rho}^{m} \backslash R_{p}$, the Lebesgue measure of which is at most $\exp (-\sqrt{p} / 4)$ on $\Omega_{p}$. For any $C=I^{1} \times \cdots \times I^{m} \in \mathscr{G}_{p}^{2}$ of order $2 p$, each $I^{i}$ contains a set $S\left(x^{i}, c_{1} 2^{-2 p}\right)$ for some $x^{i}$ and the set has Lebesgue measure $K 2^{-2 p Q}$, so $\Omega_{p}$ is contained in the event $\widetilde{\Omega}_{p}$ that the cardinality of $\mathscr{G}_{p}^{2}$ is at most $K 2^{2 p m Q} \exp (-\sqrt{p} / 4)$.

Recall that both $\mathscr{G}_{p}^{1}$ and $\mathscr{G}_{p}^{2}$ depend on $\Sigma_{1}$. We see that $\widetilde{\Omega}_{p}$ belongs to the $\sigma$-algebra $\Sigma_{1}$. Hence for $p \geq p_{0}$,

$$
\begin{aligned}
\mathrm{E}\left(X_{p}\right) & \leq \mathrm{E}\left(\mathbf{1}_{\widetilde{\Omega}_{p}} \sum_{C \in \mathscr{C}_{p}} f\left(r_{p, C}\right) r_{p, C}^{-(m-1) d} \mathbf{1}_{\left\{C \in \mathscr{G}_{p}\right\}} \mathbf{1}_{\Omega_{p, C}}\right) \\
& =\mathrm{E}\left(\mathbf{1}_{\widetilde{\Omega}_{p}} \sum_{C \in \mathscr{C}_{p}} f\left(r_{p, C}\right) r_{p, C}^{-(m-1) d} \mathbf{1}_{\left\{C \in \mathscr{G}_{p}\right\}} \mathrm{P}\left(\Omega_{p, C} \mid \Sigma_{1}\right)\right) \\
& \leq K \mathrm{E}\left(\mathbf{1}_{\widetilde{\Omega}_{p}} \sum_{C \in \mathscr{C}_{p}} f\left(r_{p, C}\right) \mathbf{1}_{\left\{C \in \mathscr{G}_{p}\right\}}\right),
\end{aligned}
$$

where the last inequality follows from Lemma 3.8 and independence of $v^{1}$ and $v^{2}$.

Now consider any dyadic cube $C \in \mathscr{C}_{p}$ of order $q$. If $C \in \mathscr{G}_{p}^{1}$, then $f\left(r_{p, C}\right) \leq$ $K 2^{-q m Q} \leq K \lambda(C)$ (where $\lambda(\cdot)$ denotes Lebesgue measure); if $C \in \mathscr{G}_{p}^{2}$, then $f\left(r_{p, C}\right) \leq$ $K 2^{-2 p m Q} p^{m Q / 2}(\log p)^{m}$. Moreover, for $p \geq p_{0}$ the dyadic cubes in $\mathscr{G}_{p}^{1}$ are disjoint and contained in $B_{3 \rho}^{1} \times \cdots \times B_{3 \rho}^{m}$. These observations, together with (4.10) and (4.5), imply

$$
\mathrm{E}\left(X_{p}\right) \leq K \mathrm{E}\left(\sum_{C \in \mathscr{C}_{p}} \lambda(C) \mathbf{1}_{\left\{C \in \mathscr{G}_{p}^{1}\right\}}+p^{m Q / 2}(\log p)^{m} \exp (-\sqrt{p} / 4)\right) \leq K \rho^{m Q} .
$$

By Fatou's lemma, we derive $\mathrm{E}(X) \leq K \rho^{m Q}<\infty$. This is a contradiction. The proof of Theorem 2.5 is complete.

\section{Examples}

In this section we provide some examples where Theorem 2.5 is applicable. These include fractional Brownian sheets, and the solutions to systems of stochastic heat and wave equations.

\subsection{Fractional Brownian sheets}

The $(N, d)$-fractional Brownian sheet with Hurst parameter $H=\left(H_{1}, \ldots, H_{N}\right) \in$ $(0,1)^{N}$ is an $\mathbb{R}^{d}$-valued continuous Gaussian random field $\left\{v(x), x \in \mathbb{R}_{+}^{N}\right\}$ with mean zero and covariance

$$
\mathrm{E}\left(v_{j}(x) v_{\ell}(y)\right)=\delta_{j, \ell} \prod_{i=1}^{N} \frac{1}{2}\left(\left|x_{i}\right|^{2 H_{i}}+\left|y_{i}\right|^{2 H_{i}}-\left|x_{i}-y_{i}\right|^{2 H_{i}}\right) .
$$

When $N=1$, it is the fractional Brownian motion and the non-existence of multiple points in the critical dimension was proved by Talagrand [13]. So we focus on the case $N \geq 2$.

Let $\alpha \in(0,1)$ be a constant. We start with the identity that any $x \in \mathbb{R}$,

$$
|x|^{2 \alpha}=c_{\alpha}^{2} \int_{\mathbb{R}} \frac{1-\cos x \xi}{|\xi|^{2 \alpha+1}} d \xi, \quad \text { where } c_{\alpha}=\left(\int_{\mathbb{R}} \frac{1-\cos \xi}{|\xi|^{2 \alpha+1}} d \xi\right)^{-1 / 2},
$$


which can be obtained by a change of variable in the integral. It implies that for any $x, y \in \mathbb{R}$,

$$
\frac{1}{2}\left(|x|^{2 \alpha}+|y|^{2 \alpha}-|x-y|^{2 \alpha}\right)=c_{\alpha}^{2} \int_{\mathbb{R}}\left[\frac{(1-\cos x \xi)(1-\cos y \xi)}{|\xi|^{2 \alpha+1}}+\frac{\sin x \xi \sin y \xi}{|\xi|^{2 \alpha+1}}\right] d \xi .
$$

It follows that for $H \in(0,1)^{N}$ and $x, y \in \mathbb{R}_{+}^{N}$, we can write

$$
\prod_{i=1}^{N} \frac{1}{2}\left(\left|x_{i}\right|^{2 H_{i}}+\left|y_{i}\right|^{2 H_{i}}-\left|x_{i}-y_{i}\right|^{2 H_{i}}\right)=c_{H}^{2} \sum_{p \in\{0,1\}^{N}} \int_{\mathbb{R}^{N}} \prod_{i=1}^{N} \frac{f_{p_{i}}\left(x_{i} \xi_{i}\right) f_{p_{i}}\left(y_{i} \xi_{i}\right)}{\left|\xi_{i}\right|^{2 H_{i}+1}} d \xi
$$

where $f_{0}(t)=1-\cos t$ and $f_{1}(t)=\sin t$. It gives a representation for the fractional Brownian sheet: If $W_{p}, p \in\{0,1\}^{N}$, are independent $\mathbb{R}^{d}$-valued Gaussian white noises on $\mathbb{R}^{N}$ and

$$
v(x):=c_{H} \sum_{p \in\{0,1\}^{N}} \int_{\mathbb{R}^{N}} \prod_{i=1}^{N} \frac{f_{p_{i}}\left(x_{i} \xi_{i}\right)}{\left|\xi_{i}\right|^{H_{i}+1 / 2}} W_{p}(d \xi),
$$

then (a continuous modification of) $\left\{v(x), x \in \mathbb{R}_{+}^{N}\right\}$ is an $(N, d)$-fractional Brownian sheet with Hurst index $H$. In particular, when $H_{i}=\frac{1}{2}$ for $i=1, \ldots, k$, the Gaussian random field $\left\{v(x), x \in \mathbb{R}^{N}\right\}$ is the Brownian sheet and (5.2) provides a harmonizable representation for it.

It is known [15] that for any compact interval $I$ in $(0, \infty)^{N}$, there exist positive finite constants $c_{1}$ and $c_{2}$ such that for all $x, y \in I$,

$$
c_{1} \sum_{j=1}^{N}\left|x_{j}-y_{j}\right|^{2 H_{j}} \leq \mathrm{E}\left(|v(x)-v(y)|^{2}\right) \leq c_{2} \sum_{j=1}^{N}\left|x_{j}-y_{j}\right|^{2 H_{j}} .
$$

We take $T=(0, \infty)^{N}$ [since $v(x)=0$ for all $x \in \partial \mathbb{R}_{+}^{N}$ a.s., the existence of multiple points is trivial on $\partial \mathbb{R}_{+}^{N}$ ]. In Lemmas $5.1-5.3$ below, we use the representation (5.2) to show that the fractional Brownian sheet satisfies the assumptions of Theorem 2.5 on $T$.

Define the random field $\left\{v(A, x), A \in \mathscr{B}\left(\mathbb{R}_{+}\right), x \in T\right\}$ by

$$
v(A, x)=c_{H} \sum_{p \in\{0,1\}^{N}} \int_{\left\{\max _{i}\left|\xi_{i}\right|^{H_{i}} \in A\right\}} \prod_{i=1}^{N} \frac{f_{p_{i}}\left(x_{i} \xi_{i}\right)}{\left|\xi_{i}\right|^{H_{i}+1 / 2}} W_{p}(d \xi) .
$$

Lemma 5.1. For any $n>1$, let $F_{n}=[1 / n, n]^{N}, a_{0}=0$ and $\gamma_{i}=H_{i}^{-1}-1$. There is a constant $c_{0}>0$ depending on $n$ such that for all $0 \leq a<b \leq \infty$ and $x, y \in F_{n}$,

$$
\|(v(x)-v([a, b), x))-(v(y)-v([a, b), y))\|_{L^{2}} \leq c_{0}\left(\sum_{i=1}^{N} a^{\gamma_{i}}\left|x_{i}-y_{i}\right|+b^{-1}\right) .
$$

Proof. Without loss of generality, we may assume $d=1$. For any $0 \leq a<b \leq \infty$, let $B=\left\{\xi \in \mathbb{R}^{N}: \max _{i}\left|\xi_{i}\right|^{H_{i}} \in[a, b)\right\}$. Then we can express its complement as

$$
\mathbb{R}^{N} \backslash B=\left\{\left|\xi_{k}\right|<a_{k}, \forall 1 \leq k \leq N\right\} \cup \bigcup_{k=1}^{N}\left\{\left|\xi_{k}\right| \geq b_{k}\right\}
$$

where $a_{i}=a^{1 / H_{i}}$ and $b_{i}=b^{1 / H_{i}}$.

Note that

$$
\begin{aligned}
& \prod_{i=1}^{N} \frac{f_{p_{i}}\left(x_{i} \xi_{i}\right)}{\left|\xi_{i}\right|^{H_{i}+1 / 2}}-\prod_{i=1}^{N} \frac{f_{p_{i}}\left(y_{i} \xi_{i}\right)}{\left|\xi_{i}\right|^{H_{i}+1 / 2}} \\
& =\sum_{i=1}^{N}\left(\frac{f_{p_{i}}\left(x_{i} \xi_{i}\right)-f_{p_{i}}\left(y_{i} \xi_{i}\right)}{\left|\xi_{i}\right|^{H_{i}+1 / 2}} \prod_{1 \leq j<i} \frac{f_{p_{j}}\left(y_{j} \xi_{j}\right)}{\left|\xi_{j}\right|^{H_{j}+1 / 2}} \prod_{i<j \leq N} \frac{f_{p_{j}}\left(x_{j} \xi_{j}\right)}{\left|\xi_{j}\right|^{H_{j}+1 / 2}}\right) .
\end{aligned}
$$


It follows that

$$
\begin{aligned}
& \|(v(x)-v([a, b), x))-(v(y)-v([a, b), y))\|_{L^{2}} \\
& \leq c_{H} \sum_{p \in\{0,1\}^{N}}\left[\int_{\left\{\left|\xi_{k}\right|<a_{k}, \forall k\right\}}\left(\prod_{i=1}^{N} \frac{f_{p_{i}}\left(x_{i} \xi_{i}\right)}{\left|\xi_{i}\right|^{H_{i}+1 / 2}}-\prod_{i=1}^{N} \frac{f_{p_{i}}\left(y_{i} \xi_{i}\right)}{\left|\xi_{i}\right|^{H_{i}+1 / 2}}\right)^{2} d \xi\right]^{1 / 2} \\
& \quad+c_{H} \sum_{p \in\{0,1\}^{N}} \sum_{k=1}^{N}\left[\int_{\left\{\left|\xi_{k}\right| \geq b_{k}\right\}}\left(\prod_{i=1}^{N} \frac{f_{p_{i}}\left(x_{i} \xi_{i}\right)}{\left|\xi_{i}\right|^{H_{i}+1 / 2}}-\prod_{i=1}^{N} \frac{f_{p_{i}}\left(y_{i} \xi_{i}\right)}{\left|\xi_{i}\right|^{H_{i}+1 / 2}}\right)^{2} d \xi\right]^{1 / 2} \\
& \leq c_{H} \sum_{p} \sum_{i=1}^{N}\left[\int_{\left\{\left|\xi_{k}\right|<a_{k}, \forall k\right\}}\left(\frac{f_{p_{i}}\left(x_{i} \xi_{i}\right)-f_{p_{i}}\left(y_{i} \xi_{i}\right)}{\left|\xi_{i}\right|^{H_{i}+1 / 2}} \prod_{1 \leq j<i} \frac{f_{p_{j}}\left(y_{j} \xi_{j}\right)}{\left|\xi_{j}\right|^{H_{j}+1 / 2}} \prod_{i<j \leq N} \frac{f_{p_{j}}\left(x_{j} \xi_{j}\right)}{\left|\xi_{j}\right|^{H_{j}+1 / 2}}\right)^{2} d \xi\right]^{1 / 2} \\
& +c_{H} \sum_{p} \sum_{i=1}^{N} \sum_{k=1}^{N}\left[\int_{\left\{\left|\xi_{k}\right| \geq b_{k}\right\}}\left(\frac{f_{p_{i}}\left(x_{i} \xi_{i}\right)-f_{p_{i}}\left(y_{i} \xi_{i}\right)}{\left|\xi_{i}\right|^{H_{i}+1 / 2}} \prod_{1 \leq j<i} \frac{f_{p_{j}}\left(y_{j} \xi_{j}\right)}{\left|\xi_{j}\right|^{H_{j}+1 / 2}} \prod_{i<j \leq N} \frac{f_{p_{j}}\left(x_{j} \xi_{j}\right)}{\left|\xi_{j}\right|^{H_{j}+1 / 2}}\right)^{2} d \xi\right]^{1 / 2} .
\end{aligned}
$$

Using the bounds $\left|f_{p_{i}}(x \xi)-f_{p_{i}}(y \xi)\right| \leq|x-y||\xi|$ and $\left|f_{p_{i}}(x \xi)-f_{p_{i}}(y \xi)\right| \leq 2$ for $p_{i}=0,1$, we see that the above is at most

$$
\begin{aligned}
c_{H} \sum_{p} \sum_{i=1}^{N}\left[\int_{\left\{\left|\xi_{i}\right|<a_{i}\right\}} \frac{\left|x_{i}-y_{i}\right|^{2}}{\left|\xi_{i}\right|^{2 H_{i}-1}}\left(\prod_{1 \leq j<i} \frac{f_{p_{j}}\left(y_{j} \xi_{j}\right)}{\left|\xi_{j}\right|^{H_{j}+1 / 2}} \prod_{i<j \leq N} \frac{f_{p_{j}}\left(x_{j} \xi_{j}\right)}{\left|\xi_{j}\right|^{H_{j}+1 / 2}}\right)^{2} d \xi\right]^{1 / 2} \\
+c_{H} \sum_{p} \sum_{i=1}^{N}\left[\int_{\left\{\left|\xi_{i}\right| \geq b_{i}\right\}} \frac{4}{\left|\xi_{i}\right|^{2 H_{i}+1}}\left(\prod_{1 \leq j<i} \frac{f_{p_{j}}\left(y_{j} \xi_{j}\right)}{\left|\xi_{j}\right|^{H_{j}+1 / 2}} \prod_{i<j \leq N} \frac{f_{p_{j}}\left(x_{j} \xi_{j}\right)}{\left|\xi_{j}\right|^{H_{j}+1 / 2}}\right)^{2} d \xi\right]^{1 / 2} \\
+c_{H} \sum_{p} \sum_{i=1}^{N} \sum_{k \neq i}\left[\int_{\left\{\left|\xi_{k}\right| \geq b_{k}\right\}} \frac{1}{\left|\xi_{k}\right|^{2 H_{k}+1}}\right. \\
\left.\times\left(\frac{f_{p_{i}}\left(x_{i} \xi_{i}\right)-f_{p_{i}}\left(y_{i} \xi_{i}\right)}{\left|\xi_{i}\right|^{H_{i}+1 / 2}} \prod_{j<i, j \neq k} \frac{f_{p_{j}}\left(y_{j} \xi_{j}\right)}{\left|\xi_{j}\right|^{H_{j}+1 / 2}} \prod_{j>i, j \neq k} \frac{f_{p_{j}}\left(x_{j} \xi_{j}\right)}{\left|\xi_{j}\right|^{H_{j}+1 / 2}}\right)^{2} d \xi\right]^{1 / 2} .
\end{aligned}
$$

Then by (5.1) and (5.3) applied on $\mathbb{R}_{+}^{N-1}$, the above sum is bounded from above by

$$
\begin{aligned}
& K \sum_{p} \sum_{i=1}^{N}\left[a_{i}^{2-2 H_{i}}\left|x_{i}-y_{i}\right|^{2} \prod_{1 \leq j<i}\left|y_{j}\right|^{2 H_{j}} \prod_{i<j \leq N}\left|x_{j}\right|^{2 H_{j}}\right]^{1 / 2} \\
& +K \sum_{p} \sum_{i=1}^{N}\left[b_{i}^{-2 H_{i}} \prod_{1 \leq j<i}\left|y_{j}\right|^{2 H_{j}} \prod_{i<j \leq N}\left|x_{j}\right|^{2 H_{j}}\right]^{1 / 2}+K \sum_{p} \sum_{i=1}^{N} \sum_{k \neq i}\left[b_{k}^{-2 H_{k}}\left|x_{i}-y_{i}\right|^{2 H_{i}}\right]^{1 / 2} .
\end{aligned}
$$

Since $\left|x_{j}\right|,\left|y_{j}\right| \leq n$, we obtain (5.4) for some $c_{0}$ depending on $n$.

Lemma 5.2. Let $F_{n}=[1 / n, n]^{N}, n>1$. Take $\varepsilon_{0}=1 /(2 n), c=3$ and $c^{\prime}=6$. Consider $x \in F_{n}, 0<\rho \leq \varepsilon_{0}$ and a compact interval $B_{3 \rho}(x) \subset F_{n}$. Let $x^{\prime}=x$. There is a finite constant $C$ such that for all $y, \bar{y} \in B_{2 \rho}(x)$, and also for all $y, \bar{y} \in B_{2 \rho}(\tilde{x}) \subset F_{n}$ with $\Delta(x, \tilde{x}) \geq 6 \rho$,

$$
\left|\mathrm{E}\left(\left(v_{j}(y)-v_{j}(\bar{y})\right) v_{j}\left(x^{\prime}\right)\right)\right| \leq C \sum_{i=1}^{N}\left|y_{i}-\bar{y}_{i}\right|^{\delta_{i}}
$$

for all $j \in\{1, \ldots, d\}$, where $\delta_{i}=\min \left\{2 H_{i}, 1\right\}$. 
Proof. Let $0<\rho<\varepsilon_{0}$. It suffices to show that there is a finite constant $C$ such that for all $y, \bar{y} \in F_{n}$,

$$
\left|\prod_{i=1}^{N}\left(\left|x_{i}\right|^{2 H_{i}}+\left|y_{i}\right|^{2 H_{i}}-\left|x_{i}-y_{i}\right|^{2 H_{i}}\right)-\prod_{i=1}^{N}\left(\left|x_{i}\right|^{2 H_{i}}+\left|\bar{y}_{i}\right|^{2 H_{i}}-\left|x_{i}-\bar{y}_{i}\right|^{2 H_{i}}\right)\right| \leq C \sum_{i=1}^{N}\left|y_{i}-\bar{y}_{i}\right|^{\delta_{i}} .
$$

For $1 \leq \ell \leq N$, let $A_{\ell}=U_{\ell}-V_{\ell}$, where

$$
U_{\ell}=\prod_{i=1}^{\ell}\left(\left|x_{i}\right|^{2 H_{i}}+\left|y_{i}\right|^{2 H_{i}}-\left|x_{i}-y_{i}\right|^{2 H_{i}}\right), \quad V_{\ell}=\prod_{i=1}^{\ell}\left(\left|x_{i}\right|^{2 H_{i}}+\left|\bar{y}_{i}\right|^{2 H_{i}}-\left|x_{i}-\bar{y}_{i}\right|^{2 H_{i}}\right) .
$$

When $\ell=1$, we have $\left|A_{1}\right| \leq\left.|| y_{1}\right|^{2 H_{1}}-\left|\bar{y}_{1}\right|^{2 H_{1}}|+|\left|x_{1}-y_{1}\right|^{2 H_{1}}-\left|x_{1}-\bar{y}_{1}\right|^{2 H_{1}} \mid$. If $2 H_{1} \leq 1$, then by the triangle inequality and the inequality $(a+b)^{2 H} \leq a^{2 H}+b^{2 H}$ for $a, b \geq 0$, we have $\left|A_{1}\right| \leq 2\left|y_{1}-\bar{y}_{1}\right|^{2 H_{1}}$; if $2 H_{1}>1$, then we can use the mean value theorem to get $\left|A_{1}\right| \leq 2 H_{1} n^{2 H_{1}-1}\left|y_{1}-\bar{y}_{1}\right|$. Thus $\left|A_{1}\right| \leq K\left|y_{1}-\bar{y}_{1}\right|^{\delta_{1}}$ for either case. For $2 \leq \ell \leq N$,

$$
\begin{aligned}
A_{\ell}= & U_{\ell-1}\left(\left|x_{\ell}\right|^{2 H_{\ell}}+\left|y_{\ell}\right|^{2 H_{\ell}}-\left|x_{\ell}-y_{\ell}\right|^{2 H_{\ell}}\right)-V_{\ell-1}\left(\left|x_{\ell}\right|^{2 H_{\ell}}+\left|\bar{y}_{\ell}\right|^{2 H_{\ell}}-\left|x_{\ell}-\bar{y}_{\ell}\right|^{2 H_{\ell}}\right) \\
= & A_{\ell-1}\left(\left|x_{\ell}\right|^{2 H_{\ell}}+\left|y_{\ell}\right|^{2 H_{\ell}}-\left|x_{\ell}-y_{\ell}\right|^{2 H_{\ell}}\right) \\
& +V_{\ell-1}\left(\left|y_{\ell}\right|^{2 H_{\ell}}-\left|\bar{y}_{\ell}\right|^{2 H_{\ell}}+\left|x_{\ell}-\bar{y}_{\ell}\right|^{2 H_{\ell}}-\left|x_{\ell}-y_{\ell}\right|^{2 H_{\ell}}\right) .
\end{aligned}
$$

Then $\left|A_{\ell}\right| \leq K\left(\left|A_{\ell-1}\right|+\left|y_{\ell}-\bar{y}_{\ell}\right|^{\delta_{\ell}}\right)$. By induction, $\left|A_{N}\right| \leq C \sum_{\ell=1}^{N}\left|y_{\ell}-\bar{y}_{\ell}\right|^{\delta_{\ell}}$.

The following lemma verifies Assumption 2.3 for fractional Brownian sheets. The sectorial local nondeterminism in Theorem 1 of $\mathrm{Wu}$ and Xiao [15] provides more information on the conditional variances among $v\left(x_{1}\right), \ldots, v\left(x_{m}\right)$.

Lemma 5.3. If $x^{1}, \ldots, x^{m} \in(0, \infty)^{N}$ are distinct points, then the random variables $v\left(x_{1}\right), \ldots, v\left(x_{m}\right)$ are linearly independent.

Proof. Suppose that $a_{1}, \ldots, a_{m}$ are real numbers such that $\sum_{\ell=1}^{m} a_{\ell} v\left(x^{\ell}\right)=0$ a.s. Recalling the representation (5.2) for $v(x)$, we have

$$
0=\mathrm{E}\left(\sum_{\ell=1}^{m} a_{\ell} v\left(x^{\ell}\right)\right)^{2}=c_{H}^{2} \sum_{p \in\{0,1\}^{N}} \int_{\mathbb{R}^{N}}\left(\sum_{\ell=1}^{m} a_{\ell} \prod_{j=1}^{N} \frac{f_{p_{j}}\left(x_{j}^{\ell} \xi_{j}\right)}{\left|\xi_{j}\right|^{H_{j}+1 / 2}}\right)^{2} d \xi
$$

Then for each $p \in\{0,1\}^{N}, \sum_{\ell=1}^{m} a_{\ell} \prod_{j=1}^{N} f_{p_{j}}\left(x_{\tilde{f}^{\ell}}^{\ell} \xi_{j}\right)=0$ and hence $\sum_{\ell=1}^{m} a_{\ell} \prod_{j=1}^{N} \tilde{f}_{p_{j}}\left(x_{j}^{\ell} \xi_{j}\right)=$ 0 for all $\xi \in \mathbb{R}^{N}$, where $\tilde{f}_{0}(t)=1-\cos t$ and $\tilde{f}_{1}(t)=-i \sin t$. It follows that

$$
\sum_{\ell=1}^{m} a_{\ell} \prod_{j=1}^{N}\left(1-\exp \left(i x_{j}^{\ell} \xi_{j}\right)\right)=\sum_{p \in\{0,1\}^{N}} \sum_{\ell=1}^{m} a_{\ell} \prod_{j=1}^{N} \tilde{f}_{p_{j}}\left(x_{j}^{\ell} \xi_{j}\right)=0
$$

for all $\xi \in \mathbb{R}^{N}$. We claim that $a_{1}=0$. Let $L_{1,1}, \ldots, L_{1, k_{1}}$ be partitions of $\{1, \ldots, m\}$ obtained from the equivalence classes of the equivalence relation $\sim_{1}$ defined by $\ell \sim_{1} k$ if and only if $x_{1}^{\ell}=x_{1}^{k}$. We may assume $1 \in L_{1,1}$. Let $\hat{x}_{1}^{1}, \ldots, \hat{x}_{1}^{m_{1}}$ be such that $x_{1}^{\ell}=\hat{x}_{1}^{k}$ for all $\ell \in L_{1, k}, k=1, \ldots, m_{1}$. Let $\xi_{2}, \ldots, \xi_{N} \in \mathbb{R}$ be arbitrary and define $c_{1,1}, c_{1,2}, \ldots, c_{1, m_{1}}$ by

$$
c_{1, k}=\sum_{\ell \in L_{1, k}} a_{\ell} \prod_{j=2}^{N}\left(1-\exp \left(i x_{j}^{\ell} \xi_{j}\right)\right) .
$$

Then by (5.5), we have

$$
c_{1,1} \exp \left(i \hat{x}_{1}^{1} \xi_{1}\right)+\cdots+c_{1, m_{1}} \exp \left(i \hat{x}_{1}^{m_{1}} \xi_{1}\right)+\left(c_{1,1}+\cdots+c_{1, m_{1}}\right)=0
$$


for all $\xi_{1} \in \mathbb{R}$. Since $\hat{x}_{1}^{1}, \ldots, \hat{x}_{1}^{m_{1}}$ are non-zero and distinct, the functions $\exp \left(i \hat{x}_{1}^{1} \xi\right), \ldots$, $\exp \left(i \hat{x}_{1}^{m_{1}} \xi\right)$ and 1 are linearly independent over $\mathbb{C}$, we have $c_{1,1}=\cdots=c_{1, m_{1}}=0$. In particular, we have

$$
\sum_{\ell \in L_{1,1}} a_{\ell} \prod_{j=2}^{N}\left(1-\exp \left(i x_{j}^{\ell} \xi_{j}\right)\right)=0
$$

for all $\xi_{2}, \ldots, \xi_{N} \in \mathbb{R}$. Next we consider the partitions $L_{2,1}, \ldots, L_{2, m_{2}}$ of $\{1, \ldots, m\}$ obtained from equivalence classes of $\sim_{2}$ defined by $\ell \sim_{2} k$ iff $x_{2}^{\ell}=x_{2}^{k}$ (with $1 \in L_{2,1}$ ). Then the argument above yields

$$
\sum_{\ell \in L_{1,1} \cap L_{2,1}} a_{\ell} \prod_{j=3}^{N}\left(1-\exp \left(i x_{j}^{\ell} \xi_{j}\right)\right)=0 .
$$

By induction, we obtain

$$
\sum_{\ell \in L_{1,1} \cap \cdots \cap L_{N, 1}} a_{\ell}=0 .
$$

Note that $L_{1,1} \cap \cdots \cap L_{N, 1}=\{1\}$ because $x^{1}, \ldots, x^{m}$ are distinct. Hence $a_{1}=0$. Similarly, we can show that $a_{\ell}=0$ for $\ell=2, \ldots, m$.

Theorem 5.4. Let $v=\left\{v(x), x \in \mathbb{R}_{+}^{N}\right\}$ be an $(N, d)$-fractional Brownian sheet with Hurst parameter $H \in(0,1)^{N}$. If $m Q \leq(m-1) d$ where $Q=\sum_{i=1}^{N} H_{i}^{-1}$, then $v$ has no $m$-multiple points on $(0, \infty)^{N}$ almost surely.

Proof. By the three lemmas above, $\left\{v(x), x \in[1 / n, n]^{N}\right\}$ satisfies the assumptions of Theorem 2.5 with $Q=\sum_{i=1}^{N} H_{i}^{-1}$ for every $n \geq 1$. Hence the result follows immediately from the theorem.

We remark that for the case of Brownian sheet, i.e., $H_{i}=1 / 2$ for all $i$, the above result provides an alternative proof for the main results in $[3,4]$.

\subsection{System of stochastic heat equations}

Let $k \geq 1$ and $\beta \in(0, k \wedge 2)$, or $k=1=\beta$. Consider the $\mathbb{R}^{d}$-valued random field $\left\{v(t, x),(t, x) \in \mathbb{R}_{+} \times \mathbb{R}^{k}\right\}$ defined by

$$
v(t, x)=\int_{\mathbb{R}} \int_{\mathbb{R}^{k}} e^{-i \xi \cdot x} \frac{e^{-i \tau t}-e^{-t|\xi|^{2}}}{|\xi|^{2}-i \tau}|\xi|^{-(k-\beta) / 2} W(d \tau, d \xi),
$$

where $W$ is a $\mathbb{C}^{d}$-valued space-time Gaussian white noise on $\mathbb{R}^{1+k}$, i.e., $W=W_{1}+i W_{2}$ and $W_{1}, W_{2}$ are independent $\mathbb{R}^{d}$-valued space-time Gaussian white noises on $\mathbb{R}^{1+k}$. According to Proposition 7.2 of [5], the process $\hat{v}(t, x):=\operatorname{Re} v(t, x),(t, x) \in \mathbb{R}_{+} \times \mathbb{R}^{k}$, has the same law as the mild solution to the system of stochastic heat equations on $\mathbb{R}_{+} \times \mathbb{R}^{k}$ :

$$
\left\{\begin{array}{l}
\frac{\partial}{\partial t} \hat{v}_{j}(t, x)=\Delta \hat{v}_{j}(t, x)+\dot{\hat{W}}_{j}(t, x), \quad j=1, \ldots, d \\
\hat{v}(0, x)=0
\end{array}\right.
$$

where $\hat{W}$ is an $\mathbb{R}^{d}$-valued spatially homogeneous Gaussian noise that is white in time with spatial covariance $|x-y|^{-\beta}$ if $k \geq 1$ and $\beta \in(0, k \wedge 2)$; it is an $\mathbb{R}^{d}$-valued space-time Gaussian white noise when $k=1=\beta$. We take $T=(0, \infty) \times \mathbb{R}^{k}$.

By Lemma 7.3 of [5], Assumption 2.1 is satisfied with

$$
\gamma_{1}=\frac{2+\beta}{2-\beta}, \quad \gamma_{2}=\cdots=\gamma_{k+1}=\frac{\beta}{2-\beta},
$$


and

In this case,

$$
\alpha_{1}=\frac{2-\beta}{4}, \quad \alpha_{2}=\cdots=\alpha_{k+1}=\frac{2-\beta}{2}
$$

$$
\Delta((t, x),(s, y))=|t-s|^{\frac{2-\beta}{4}}+|x-y|^{\frac{2-\beta}{2}} .
$$

In the following lemma, we verify Assumption 2.2 for the Gaussian random field $\hat{v}$.

Lemma 5.5. Let $k \geq 1, \beta \in(0, k \wedge 2)$, or $k=1=\beta$. Let $F \subset(0, \infty) \times \mathbb{R}^{k}$ be a compact interval. Let $c=3, c^{\prime}=6$ and $0<\varepsilon_{0}<1$ be any small number. For any $(t, x) \in F$ and $0<\rho \leq \varepsilon_{0}$ with $B_{3 \rho}(t, x) \subset F$, let $\left(t^{\prime}, x^{\prime}\right)=(t, x)$. Take any $\delta \in\left(\frac{2-\beta}{2},(2-\beta) \wedge 1\right)$. Then there exists a finite constant $C$ such that for all $\left(s_{1}, y_{1}\right),\left(s_{2}, y_{2}\right) \in B_{2 \rho}(t, x)$, and also for all $\left(s_{1}, y_{1}\right),\left(s_{2}, y_{2}\right) \in B_{2 \rho}(\tilde{t}, \tilde{x}) \subset F$ with $\Delta((t, x),(\tilde{t}, \tilde{x})) \geq 6 \rho$, for all $j \in\{1, \ldots, d\}$,

$$
\left|\mathrm{E}\left[\left(\hat{v}_{j}\left(s_{1}, y_{1}\right)-\hat{v}_{j}\left(s_{2}, y_{2}\right)\right) \hat{v}_{j}\left(t^{\prime}, x^{\prime}\right)\right]\right| \leq C\left(\left|s_{1}-s_{2}\right|^{\frac{2-\beta}{2}}+\left|y_{1}-y_{2}\right|^{\delta}\right) .
$$

Proof. Suppose that $\left(s_{1}, y_{1}\right),\left(s_{2}, y_{2}\right) \in B_{2 \rho}(t, x)$ or $\left(s_{1}, y_{1}\right),\left(s_{2}, y_{2}\right) \in B_{2 \rho}(\tilde{t}, \tilde{x}) \subset F$ with $\Delta((t, x),(\tilde{t}, \tilde{x})) \geq 6 \rho$. Recall that

$$
\hat{v}(t, x)=\operatorname{Re} \int_{\mathbb{R}} \int_{\mathbb{R}^{k}} e^{-i \xi \cdot x} \frac{e^{-i \tau t}-e^{-t|\xi|^{2}}}{|\xi|^{2}-i \tau}|\xi|^{-(k-\beta) / 2} W(d \tau, d \xi) .
$$

Then for any $j \in\{1, \ldots, d\}$,

$$
\begin{aligned}
& \mathrm{E}\left[\left(\hat{v}_{j}\left(s_{1}, y_{1}\right)-\hat{v}_{j}\left(s_{2}, y_{2}\right)\right) \hat{v}_{j}\left(t^{\prime}, x^{\prime}\right)\right] \\
& =\operatorname{Re} \int_{\mathbb{R}} \int_{\mathbb{R}^{k}}\left(e^{-i \xi \cdot y_{1}} \frac{e^{-i \tau s_{1}}-e^{-s_{1}|\xi|^{2}}}{|\xi|^{2}-i \tau}-e^{-i \xi \cdot y_{2}} \frac{e^{-i \tau s_{2}}-e^{-s_{2}|\xi|^{2}}}{|\xi|^{2}-i \tau}\right) \\
& \times \overline{\left(e^{-i \xi \cdot x^{\prime}} \frac{e^{-i \tau t^{\prime}}-e^{-t^{\prime}|\xi|^{2}}}{|\xi|^{2}-i \tau}\right)} \frac{d \tau d \xi}{|\xi|^{k-\beta}} \\
& =I_{1}+I_{2},
\end{aligned}
$$

where

$$
I_{1}=\operatorname{Re} \int_{\mathbb{R}} \int_{\mathbb{R}^{k}}\left(e^{-i \xi \cdot\left(y_{1}-x^{\prime}\right)}-e^{-i \xi \cdot\left(y_{2}-x^{\prime}\right)}\right) \frac{\left(e^{-i \tau s_{1}}-e^{-s_{1}|\xi|^{2}}\right)\left(e^{i \tau t^{\prime}}-e^{-t^{\prime}|\xi|^{2}}\right)}{\left(|\xi|^{4}+\tau^{2}\right)|\xi|^{k-\beta}} d \tau d \xi
$$

and

$$
I_{2}=\operatorname{Re} \int_{\mathbb{R}} \int_{\mathbb{R}^{k}} e^{-i \xi \cdot\left(y_{2}-x^{\prime}\right)} \frac{\left(e^{-i \tau s_{1}}-e^{-i \tau s_{2}}-e^{-s_{1}|\xi|^{2}}+e^{-s_{2}|\xi|^{2}}\right)\left(e^{i \tau t^{\prime}}-e^{-t^{\prime}|\xi|^{2}}\right)}{\left(|\xi|^{4}+\tau^{2}\right)|\xi|^{k-\beta}} d \tau d \xi .
$$

Let us first consider $I_{1}$. We can write

$$
e^{i \tau t^{\prime}}-e^{-t^{\prime}|\xi|^{2}}=\left(e^{i \tau t^{\prime}}-1\right)+\left(1-e^{-t^{\prime}|\xi|^{2}}\right) .
$$

It follows that $\left|I_{1}\right| \leq J_{1}+J_{1}^{\prime}$, where

$$
J_{1}=\int_{\mathbb{R}} \int_{\mathbb{R}^{k}}\left|e^{-i \xi \cdot\left(y_{1}-x^{\prime}\right)}-e^{-i \xi \cdot\left(y_{2}-x^{\prime}\right)}\right| \frac{2\left|e^{i \tau t^{\prime}}-1\right|}{\left(|\xi|^{4}+\tau^{2}\right)|\xi|^{k-\beta}} d \tau d \xi
$$

and

$$
J_{1}^{\prime}=\int_{\mathbb{R}} \int_{\mathbb{R}^{k}}\left|e^{-i \xi \cdot\left(y_{1}-x^{\prime}\right)}-e^{-i \xi \cdot\left(y_{2}-x^{\prime}\right)}\right| \frac{2\left(1-e^{-t^{\prime}|\xi|^{2}}\right)}{\left(|\xi|^{4}+\tau^{2}\right)|\xi|^{k-\beta}} d \tau d \xi .
$$

Choose and fix any $\delta \in\left(\frac{2-\beta}{2},(2-\beta) \wedge 1\right)$. We have the following elementary inequalities:

$$
\left|e^{i z}-1\right| \leq 2 \wedge|z| \leq 2^{1-\delta}|z|^{\delta} \quad \text { for all } z \in \mathbb{R}
$$




$$
\text { and }\left|e^{-x}-e^{-y}\right| \leq 1 \wedge|x-y| \text { for all } x, y \geq 0 .
$$

For $J_{1}$, using these inequalities and passing to polar coordinates $r=|\xi|$, we get that

$$
J_{1}=\int_{\mathbb{R}} d \tau \int_{0}^{\infty} d r 2^{1-\delta} r^{\delta}\left|y_{1}-y_{2}\right|^{\delta} \frac{C(1 \wedge|\tau|)}{\left(r^{4}+\tau^{2}\right) \mid r^{1-\beta}}
$$

where $C$ is a constant depending on $k$ and $F$. For fixed $\tau$, we use the change of variable $r=|\tau|^{1 / 2} \tilde{r}$ to deduce that

$$
J_{1} \leq C\left|y_{1}-y_{2}\right|^{\delta} \int_{\mathbb{R}} d \tau \frac{1 \wedge|\tau|}{\tau^{1+\frac{2-\beta-\delta}{2}}} \int_{0}^{\infty} \frac{d \tilde{r}}{\left(\tilde{r}^{4}+1\right) \tilde{r}^{1-\beta-\delta}}
$$

Since the integrals in $\tau$ and $\tilde{r}$ are both finite, we have $J_{1} \leq C\left|y_{1}-y_{2}\right|^{\delta}$, where the constant $C$ depends on $k, F, \beta$ and $\delta$.

For $J_{1}^{\prime}$, we use (5.8) and (5.9), pass to polar coordinates $r=|\xi|$, and then for fixed $r$, use the change of variable $\tau=r^{2} \tilde{\tau}$ to get

$$
J_{1}^{\prime} \leq C\left|y_{1}-y_{2}\right|^{\delta} \int_{0}^{\infty} \frac{\left(1 \wedge r^{2}\right) d r}{r^{3-\beta-\delta}} \int_{\mathbb{R}} \frac{d \tilde{\tau}}{1+\tilde{\tau}^{2}}
$$

Note that the integrals in $r$ and $\tilde{\tau}$ are both finite. Hence, we deduce that $\left|I_{1}\right| \leq C\left|y_{1}-y_{2}\right|^{\delta}$.

For the other integral $I_{2}$, we have $\left|I_{2}\right| \leq J_{2}+J_{2}^{\prime}$, where

$$
J_{2}=\int_{\mathbb{R}} \int_{\mathbb{R}^{k}} \frac{2\left|e^{-i \tau s_{1}}-e^{-i \tau s_{2}}\right|}{\left(|\xi|^{4}+\tau^{2}\right)|\xi|^{k-\beta}} d \tau d \xi \quad \text { and } \quad J_{2}^{\prime}=\int_{\mathbb{R}} \int_{\mathbb{R}^{k}} \frac{2\left|e^{-s_{1}|\xi|^{2}}-e^{-s_{2}|\xi|^{2}}\right|}{\left(|\xi|^{4}+\tau^{2}\right)|\xi|^{k-\beta}} d \tau d \xi
$$

For $J_{2}$, use the first inequality in (5.8) and use polar coordinates to get that

$$
J_{2} \leq C \int_{\mathbb{R}} d \tau \int_{0}^{\infty} d r \frac{2 \wedge|\tau|\left|s_{1}-s_{2}\right|}{\left(r^{4}+\tau^{2}\right) r^{1-\beta}} .
$$

For $\tau$ fixed, by changing variable $r=|\tau|^{1 / 2} \tilde{r}$ in the second integral,

$$
J_{2} \leq C \int_{\mathbb{R}} d \tau \frac{2 \wedge|\tau|\left|s_{1}-s_{2}\right|}{|\tau|^{2-\frac{\beta}{2}}} \int_{0}^{\infty} \frac{d \tilde{r}}{\tilde{r}^{4}+1}
$$

The integral in $\tilde{r}$ is finite. Then splitting the integral in $\tau$ into two parts where $|\tau| \leq \frac{2}{\left|s_{1}-s_{2}\right|}$ and $|\tau|>\frac{2}{\left|s_{1}-s_{2}\right|}$ leads to

$$
J_{2} \leq C \int_{|\tau| \leq \frac{2}{\left|s_{1}-s_{2}\right|}} \frac{|\tau|\left|s_{1}-s_{2}\right|}{|\tau|^{2-\frac{\beta}{2}}} d \tau+C \int_{|\tau|>\frac{2}{\left|s_{1}-s_{2}\right|}} \frac{2}{|\tau|^{2-\frac{\beta}{2}}} d \tau
$$

which implies $J_{2} \leq C\left|s_{1}-s_{2}\right|^{\frac{2-\beta}{2}}$.

For $J_{2}^{\prime}$, by using (5.9) and polar coordinates, we have

$$
J_{2}^{\prime} \leq C \int_{\mathbb{R}} d \tau \int_{0}^{\infty} d r \frac{1 \wedge r^{2}\left|s_{1}-s_{2}\right|}{\left(r^{4}+\tau^{2}\right) r^{1-\beta}}
$$

Then we can permute the integrals, use (for $r$ fixed) the change of variable $\tau=r^{2} \tilde{\tau}$ and then split the integral in $r$ into two parts where $r \leq \frac{1}{\left|s_{1}-s_{2}\right|^{1 / 2}}$ and $r>\frac{1}{\left|s_{1}-s_{2}\right|^{1 / 2}}$ to derive that $J_{2}^{\prime} \leq C\left|s_{1}-s_{2}\right|^{\frac{2-\beta}{2}}$. This completes the proof of (5.7).

The next lemma verifies Assumption 2.3 and it can also be found in [11, Lemma A.5.3]. 
Lemma 5.6. Let $\left(t^{1}, x^{1}\right), \ldots,\left(t^{m}, x^{m}\right)$ be distinct points in $(0, \infty) \times \mathbb{R}^{k}$. Then the random variables $\hat{v}_{1}\left(t^{1}, x^{1}\right), \ldots, \hat{v}_{1}\left(t^{m}, x^{m}\right)$ are linearly independent.

Proof. Suppose that $a_{1}, \ldots, a_{m}$ are real numbers such that $\sum_{j=1}^{m} a_{j} \hat{v}_{1}\left(t^{j}, x^{j}\right)=0$ a.s. Then

$$
0=\mathrm{E}\left(\sum_{j=1}^{m} a_{j} \hat{v}_{1}\left(t^{j}, x^{j}\right)\right)^{2}=\int_{\mathbb{R}} \int_{\mathbb{R}^{k}}\left|\sum_{j=1}^{m} a_{j} e^{-i \xi \cdot x^{j}}\left(e^{-i \tau t^{j}}-e^{-t^{j}|\xi|^{2}}\right)\right|^{2} \frac{d \tau d \xi}{\left(|\xi|^{4}+\tau^{2}\right)|\xi|^{k-\beta}}
$$

and thus $\sum_{j=1}^{m} a_{j} e^{-i \xi \cdot x^{j}}\left(e^{-i \tau t^{j}}-e^{-t^{j}|\xi|^{2}}\right)=0$ for all $\tau \in \mathbb{R}$ and $\xi \in \mathbb{R}^{k}$. We claim that $a_{j}=0$ for all $j=1, \ldots, m$. Let $\hat{t}^{1}, \ldots, \hat{t}^{p}$ be all distinct values of the $t^{j}$ 's. Fix an arbitrary $\xi \in \mathbb{R}^{k}$. Then for all $\tau \in \mathbb{R}$, we have

$$
\sum_{\ell=1}^{p}\left(\sum_{j: t^{j}=\hat{t}^{\ell}} a_{j} e^{-i \xi \cdot x^{j}}\right) e^{-i \tau \hat{t}^{\ell}}-\sum_{j=1}^{m} a_{j} e^{-i \xi \cdot x^{j}-t^{j}|\xi|^{2}}=0 .
$$

Since the functions $e^{-i \tau \hat{t}^{1}}, \ldots, e^{-i \tau \hat{t}^{p}}, 1$ are linearly independent over $\mathbb{C}$, it follows that for all $\xi \in \mathbb{R}^{k}$, for all $\ell=1, \ldots, p$,

$$
\sum_{j: t^{j}=\hat{t}^{\ell}} a_{j} e^{-i \xi \cdot x^{j}}=0
$$

Since $\left(t^{1}, x^{1}\right), \ldots,\left(t^{n}, x^{n}\right)$ are distinct, the $x^{j}$ 's that appear in the sum in (5.10) are distinct for any fixed $\ell$. By linear independence of the functions $e^{-i \xi \cdot x^{j}}$, we conclude that $a_{j}=0$ for all $j$.

The following result solves the existence problem of $m$-multiple points for (5.6).

Theorem 5.7. If $m(4+2 k) /(2-\beta) \leq(m-1) d$, then $\left\{\hat{v}(t, x), t \in(0, \infty), x \in \mathbb{R}^{k}\right\}$ has no m-multiple points a.s.

Proof. Assumption 2.1 is satisfied with $Q=(4+2 k) /(2-\beta)$ by Lemma 7.3 of [5]. Also, Assumptions 2.2 and 2.3 are satisfied by Lemmas 5.5 and 5.6 above. The result follows from Theorem 2.5.

\subsection{System of stochastic wave equations}

Let $k \geq 1$ and $\beta \in(1, k \wedge 2)$ or $k=1=\beta$. Consider the $\mathbb{R}^{d}$-valued random field $\left\{v(t, x),(t, x) \in \mathbb{R}_{+} \times \mathbb{R}^{k}\right\}$ defined by

$$
v(t, x)=\int_{\mathbb{R}} \int_{\mathbb{R}^{k}} F(t, x, \tau, \xi)|\xi|^{-(k-\beta) / 2} W(d \tau, d \xi),
$$

where $W$ is a $\mathbb{C}^{d}$-valued space-time Gaussian white noise on $\mathbb{R}^{1+k}$ and

$$
F(t, x, \tau, \xi)=\frac{e^{-i \xi \cdot x-i \tau t}}{2|\xi|}\left[\frac{1-e^{i t(\tau+|\xi|)}}{\tau+|\xi|}-\frac{1-e^{i t(\tau-|\xi|)}}{\tau-|\xi|}\right]
$$

By Proposition 9.2 of [5], the process $\hat{v}(t, x)=\operatorname{Re} v(t, x),(t, x) \in \mathbb{R}_{+} \times \mathbb{R}^{k}$, has the same law as the mild solution to the system of stochastic wave equations on $\mathbb{R}_{+} \times \mathbb{R}^{k}$ :

$$
\left\{\begin{array}{l}
\frac{\partial^{2}}{\partial t^{2}} \hat{v}_{j}(t, x)=\Delta \hat{v}_{j}(t, x)+\dot{\hat{W}}_{j}(t, x), \quad j=1, \ldots, d, \\
\hat{v}(0, x)=0, \quad \frac{\partial}{\partial t} \hat{v}(0, x)=0
\end{array}\right.
$$

where $\hat{W}$ is the spatially homogeneous $\mathbb{R}^{d}$-valued Gaussian noise as in (5.6). 
By Lemma 9.6 of [5], Assumption 2.1 is satisfied with $\gamma_{j}=\frac{\beta}{2-\beta}$ and $\alpha_{j}=\frac{2-\beta}{2}$ for all $j$. In this case,

$$
\Delta((t, x),(s, y))=|t-s|^{\frac{2-\beta}{2}}+|x-y|^{\frac{2-\beta}{2}} .
$$

In the following lemma, we check Assumption 2.2 for the Gaussian random field $\hat{v}$.

Lemma 5.8. Assume that $k=1=\beta$ or $1<\beta<k \wedge 2$. Let $F \subset(0, \infty) \times \mathbb{R}^{k}$ be a compact interval. Let $c=4, c^{\prime}=8$ and $0<\varepsilon_{0}<1$ be small enough such that $t-\left(4 \varepsilon_{0}\right)^{\alpha_{1}^{-1}}>0$ for all $(t, x) \in F$. For any $(t, x) \in I$ and $0<\rho \leq \varepsilon_{0}$ with $B_{4 \rho}(t, x) \subset F$, let $\left(t^{\prime}, x^{\prime}\right)=\left(t-(4 \rho)^{\alpha_{1}^{-1}}, x\right)$. Then there is a finite constant $C$ such that for all $\left(s_{1}, y_{1}\right),\left(s_{2}, y_{2}\right) \in B_{2 \rho}(t, x)$, and also for all $\left(s_{1}, y_{1}\right),\left(s_{2}, y_{2}\right) \in B_{2 \rho}(\tilde{t}, \tilde{x}) \subset F$ with $\Delta((t, x),(\tilde{t}, \tilde{x})) \geq 8 \rho$, for all $j \in\{1, \ldots, d\}$,

$$
\left|\mathrm{E}\left[\left(\hat{v}_{j}\left(s_{1}, y_{1}\right)-\hat{v}_{j}\left(s_{2}, y_{2}\right)\right) \hat{v}_{j}\left(t^{\prime}, x^{\prime}\right)\right]\right| \leq C\left(\left|s_{1}-s_{2}\right|^{2-\beta}+\left|y_{1}-y_{2}\right|^{2-\beta}\right) .
$$

Proof. Let $G$ be the fundamental solution of the wave equation.

First, consider the case $k=1=\beta$ (spatial dimension one with space-time white noise). In this case, $G(s, y)=\frac{1}{2} \mathbf{1}_{\{|y| \leq s\}}$. Consider $\left(s_{1}, y_{1}\right),\left(s_{2}, y_{2}\right) \in B_{2 \rho}(t, x)$ or $\left(s_{1}, y_{1}\right),\left(s_{2}, y_{2}\right) \in$ $B_{2 \rho}(\tilde{t}, \tilde{x}) \subset F$ with $\Delta((t, x),(\tilde{\tilde{t}}, \tilde{x})) \geq 8 \rho$. Without loss of generality, assume $s_{1} \leq s_{2}$.

Suppose $t^{\prime} \leq s_{1}$. Then

$$
\begin{aligned}
& \mathrm{E}\left[\left(\hat{v}_{j}\left(s_{1}, y_{1}\right)-\hat{v}_{j}\left(s_{2}, y_{2}\right)\right) \hat{v}_{j}\left(t^{\prime}, x^{\prime}\right)\right] \\
& =\frac{1}{4} \int_{0}^{t^{\prime}} d r \int_{\mathbb{R}} d \bar{y}\left(\mathbf{1}_{\left\{\left|\bar{y}-y_{1}\right| \leq s_{1}-r\right\}}-\mathbf{1}_{\left\{\left|\bar{y}-y_{2}\right| \leq s_{2}-r\right\}}\right) \mathbf{1}_{\left\{\left|\bar{y}-x^{\prime}\right| \leq t^{\prime}-r\right\}}
\end{aligned}
$$

and

$$
\begin{aligned}
& \left|\mathrm{E}\left[\left(\hat{v}_{j}\left(s_{1}, y_{1}\right)-\hat{v}_{j}\left(s_{2}, y_{2}\right)\right) \hat{v}_{j}\left(t^{\prime}, x^{\prime}\right)\right]\right| \\
& \leq \frac{1}{4} \int_{0}^{s_{1}} d r \int_{\mathbb{R}} d \bar{y}\left|\mathbf{1}_{\left\{\left|\bar{y}-y_{1}\right| \leq s_{1}-r\right\}}-\mathbf{1}_{\left\{\left|\bar{y}-y_{2}\right| \leq s_{2}-r\right\}}\right| .
\end{aligned}
$$

Since the value of the integrand is either 0 or 1 , it follows that

$$
\begin{aligned}
& \left|\mathrm{E}\left[\left(\hat{v}_{j}\left(s_{1}, y_{1}\right)-\hat{v}_{j}\left(s_{2}, y_{2}\right)\right) \hat{v}_{j}\left(t^{\prime}, x^{\prime}\right)\right]\right| \\
& \leq \frac{1}{4} \int_{0}^{s_{1}} d r \int_{\mathbb{R}} d \bar{y}\left|\mathbf{1}_{\left\{\left|\bar{y}-y_{1}\right| \leq s_{1}-r\right\}}-\mathbf{1}_{\left\{\left|\bar{y}-y_{2}\right| \leq s_{2}-r\right\}}\right|^{2} \\
& \leq \mathrm{E}\left|\hat{v}_{j}\left(s_{1}, y_{2}\right)-\hat{v}_{j}\left(s_{2}, y_{2}\right)\right|^{2} \\
& \leq C\left(\left|s_{1}-s_{2}\right|+\left|y_{1}-y_{2}\right|\right) .
\end{aligned}
$$

The last inequality follows from Lemma 3.1 since Assumption 2.1 is satisfied.

Suppose $s_{1}<t^{\prime}$. Then

$$
\begin{aligned}
& \mathrm{E}\left[\left(\hat{v}_{j}\left(s_{1}, y_{1}\right)-\hat{v}_{j}\left(s_{2}, y_{2}\right)\right) \hat{v}_{j}\left(t^{\prime}, x^{\prime}\right)\right] \\
& =\frac{1}{4} \int_{0}^{s_{1}} d r \int_{\mathbb{R}} d \bar{y}\left(\mathbf{1}_{\left\{\left|\bar{y}-y_{1}\right| \leq s_{1}-r\right\}}-\mathbf{1}_{\left\{\left|\bar{y}-y_{2}\right| \leq s_{2}-r\right\}}\right) \mathbf{1}_{\left\{\left|\bar{y}-x^{\prime}\right| \leq t^{\prime}-r\right\}} \\
& \quad-\frac{1}{4} \int_{s_{1}}^{s_{2} \wedge t^{\prime}} d r \int_{\mathbb{R}} d \bar{y} \mathbf{1}_{\left\{\left|\bar{y}-y_{2}\right| \leq s_{2}-r\right\}} \mathbf{1}_{\left\{\left|\bar{y}-x^{\prime}\right| \leq t^{\prime}-r\right\}} .
\end{aligned}
$$

The first integral is bounded by $C\left(\left|s_{1}-s_{2}\right|+\left|y_{1}-y_{2}\right|\right)$ by the same argument as above, and the second integral is bounded by $C\left|s_{1}-s_{2}\right|$ since the integrand is bounded by 1 and its support is also bounded over all possible $\left(s_{2}, y_{2}\right),\left(t^{\prime}, x^{\prime}\right) \in F$ by the compactness of $F$. This proves (5.11). 
For the case $1<\beta<k \wedge 2$ (colored noise), recall that $\mathcal{F} G(s, \cdot)(\xi)=\sin (s|\xi|) /|\xi|$. If $t^{\prime} \leq s_{1}$, (5.11) can be proved in exactly the same way as in Lemma 9.6 of [5]. Now suppose $s_{1}<t^{\prime}$. For time increments where $s_{1} \neq s_{2}$ and $y_{1}=y_{2}=y$, we have

$$
\begin{aligned}
& \mathrm{E}\left[\left(\hat{v}_{j}\left(s_{1}, y\right)-\hat{v}_{j}\left(s_{2}, y\right)\right) \hat{v}_{j}\left(t^{\prime}, x^{\prime}\right)\right] \\
& =\int_{0}^{s_{1}} d r \int_{\mathbb{R}^{k}} d \xi|\xi|^{\beta-2-k} e^{-i \xi \cdot\left(y-x^{\prime}\right)}\left(\sin \left(\left(s_{1}-r\right)|\xi|\right)-\sin \left(\left(s_{2}-r\right)|\xi|\right)\right) \sin \left(\left(t^{\prime}-r\right)|\xi|\right) \\
& \quad-\int_{s_{1}}^{s_{2} \wedge t^{\prime}} d r \int_{\mathbb{R}^{k}} d \xi|\xi|^{\beta-2-k} e^{-i \xi \cdot\left(y-x^{\prime}\right)} \sin \left(\left(s_{2}-r\right)|\xi|\right) \sin \left(\left(t^{\prime}-r\right)|\xi|\right) .
\end{aligned}
$$

With slight modifications of the proof of Lemma 9.6 in [5], one can show that the first integral in (5.12) is equal to

$$
\begin{aligned}
s_{1}^{3-\beta} \int_{\mathbb{R}^{k}} d \eta|\eta|^{\beta-2-k} e^{-i \eta \cdot\left(y-x^{\prime}\right)}[ & \left(\cos \left(\frac{s_{1}-t^{\prime}}{s_{1}}|\eta|\right)-\cos \left(\frac{s_{2}-t^{\prime}}{s_{1}}|\eta|\right)\right) \\
& \left.-\frac{\sin |\eta|}{|\eta|}\left(\cos \left(\frac{t^{\prime}}{s_{1}}|\eta|\right)-\cos \left(\frac{s_{2}-s_{1}+t^{\prime}}{s_{1}}|\eta|\right)\right)\right]
\end{aligned}
$$

and that it is bounded by $C\left|s_{1}-s_{2}\right|^{2-\beta}$. By $|\sin (x)| \leq|x| \wedge 1$, one can see that the second integral in (5.12) is bounded by $C\left|s_{1}-s_{2}\right|$. Hence (5.11) is satisfied for time increments. For space increments where $s_{1}=s_{2}$ and $y_{1} \neq y_{2}$, the proof of (5.11) is the same as that of Lemma 9.6 in [5].

Lemma 5.9. Let $\left(t^{1}, x^{1}\right), \ldots,\left(t^{m}, x^{m}\right)$ be distinct points in $T=(0, \infty) \times \mathbb{R}^{k}$. Then the random variables $\hat{v}_{1}\left(t^{1}, x^{1}\right), \ldots, \hat{v}_{1}\left(t^{m}, x^{m}\right)$ are linearly independent.

Proof. Suppose that $a_{1}, \ldots, a_{m}$ are real numbers such that $\sum_{j=1}^{m} a_{j} \hat{v}_{1}\left(t^{j}, x^{j}\right)=0$ a.s. Then

$$
0=\mathrm{E}\left(\sum_{j=1}^{m} a_{j} \hat{v}_{1}\left(t^{j}, x^{j}\right)\right)^{2}=\int_{\mathbb{R}} \int_{\mathbb{R}^{k}}\left|\sum_{j=1}^{m} a_{j} F\left(t^{j}, x^{j}, \tau, \xi\right)\right|^{2} \frac{d \tau d \xi}{|\xi|^{k-\beta}} .
$$

It follows that $\tau \in \mathbb{R}$ and $\xi \in \mathbb{R}^{k}, \sum_{j=1}^{m} a_{j} F\left(t^{j}, x^{j}, \tau, \xi\right)=0$ and thus

$$
\sum_{j=1}^{m} b_{j} e^{-i \tau t^{j}}+c_{1} \tau+c_{2}=0
$$

where $b_{j}=-2 a_{j}|\xi| e^{-i \xi \cdot x^{j}}$,

$$
c_{1}=-\sum_{j=1}^{m} a_{j} e^{-i \xi \cdot x^{j}}\left(e^{i t^{j}|\xi|}-e^{-i t^{j}|\xi|}\right)
$$

and

$$
c_{2}=\sum_{j=1}^{m} a_{j}|\xi| e^{-i \xi \cdot x^{j}}\left(e^{i t^{j}|\xi|}+e^{-i t^{j}|\xi|}\right) .
$$

We claim that $a_{j}=0$ for all $j=1, \ldots, m$. Let $\hat{t}^{1}, \ldots, \hat{t}^{p}$ be all distinct values of the $t^{j}$ 's. If we take arbitrary $\xi \in \mathbb{R}^{k}$ and take derivative with respect to $\tau$, we see that

$$
\sum_{\ell=1}^{p}\left(-i \hat{t}^{\ell} \sum_{j: t^{j}=\hat{t}^{\ell}} b_{j}\right) e^{-i \tau \hat{t}^{\ell}}+c_{1}=0
$$

for all $\tau \in \mathbb{R}$. Since the functions $e^{-i \tau \hat{t}^{1}}, \ldots, e^{-i \tau \hat{t}^{p}}, 1$ are linearly independent over $\mathbb{C}$, we have

$$
-i \hat{t}^{1} \sum_{j: t^{j}=\hat{t}^{\ell}} b_{j}=0
$$


for all $\ell=1, \ldots, p$. It implies that for all $\xi \in \mathbb{R}^{k}$, for all $\ell=1, \ldots, p$,

$$
\sum_{j: t^{j}=\hat{t}^{\ell}} a_{j} e^{-i \xi \cdot x^{j}}=0
$$

Since $\left(t^{1}, x^{1}\right), \ldots,\left(t^{m}, x^{m}\right)$ are distinct, the $x^{j}$ 's that appear in the sum in (5.13) are distinct for any fixed $\ell$. By linear independence of the functions $e^{-i \xi \cdot x^{j}}$, we conclude that $a_{j}=0$ for all $j$.

Theorem 5.10. Assume $k=1=\beta$ or $1<\beta<k \wedge 2$. If $m(2+2 k) /(2-\beta) \leq(m-1) d$, then $\left\{\hat{v}(t, x), t \in(0, \infty), x \in \mathbb{R}^{k}\right\}$ has no m-multiple points a.s.

Proof. Assumption 2.1 is satisfied with $Q=(2+2 k) /(2-\beta)$ by Lemma 9.3 of [5]. Assumptions 2.2 and 2.3 are satisfied by Lemmas 5.8 and 5.9 above. Hence the result follows from Theorem 2.5.

\section{References}

[1] T.W. Anderson, The integral of a symmetric unimodal function over a symmetric convex set and some probability inequalities. Proc. Amer. Math. Soc. 6 (1955), 170-176. MR-0069229

[2] R.C. Dalang, D. Khoshnevisan and E. Nualart, Hitting probabilities for systems of non-linear stochastic heat equations with additive noise. Latin Amer. J. Probab. Statist. (ALEA) 3 (2007), 231-271. MR-2365643

[3] R.C. Dalang, D. Khoshnevisan, E. Nualart, D. Wu and Y. Xiao, Critical Brownian sheet does not have double points. Ann. Probab. 40 (2012), no. 4, 1829-1859. MR-2978539

[4] R.C. Dalang and C. Mueller, Multiple points of the Brownian sheet in critical dimensions. Ann. Probab. 43 (2015), no. 4, 1577-1593. MR-3353809

[5] R.C. Dalang, C. Mueller and Y. Xiao, Polarity of points for Gaussian random fields. Ann. Probab. 45 (2017), no. 6B, 4700-4751. MR-3737922

[6] A. Goldman, Points multiples des trajectoires de processus Gaussiens. Z. Wahrsch. Verw. Gebiete. 75 (1981), 481-494. MR-0631372

[7] A. Käenmäki, T. Rajala and V. Suomala, Existence of doubling measures via generalised nested cubes. Proc. Amer. Math. Soc. 140 (2012), no. 9, 3275-3281. MR-2917099

[8] N. Kôno, Double points of a Gaussian sample path. Z. Wahrsch. Verw. Gebiete. 45 (1978), 175-180. MR-0510534

[9] M. Ledoux, Isoperimetry and Gaussian analysis. Lectures on Probability Theory and Statistics (Saint-Flour, 1994). Lecture Notes in Math. 1648 165-294. Springer, Berlin, 1996. MR1600888

[10] C. Mueller and R. Tribe, Hitting properties of a random string. Electron. J. Probab. 7 (2002), no. 10,29 pp. MR-1902843

[11] F. Pu, The stochastic heat equation: hitting probabilities and the probability density function of the supremum via Malliavin calculus. Ph.D. thesis no. 8695 (2018), Ecole Polytechnique Fédérale de Lausanne, Switzerland.

[12] J. Rosen, Self-intersections of random fields. Ann. Probab. 12 (1984), 108-119. MR-0723732

[13] M. Talagrand, Multiple points of trajectories of multiparameter fractional Brownian motion. Probab. Theory Relat. Fields 112 (1998), 545-563. MR-1664704

[14] M. Talagrand, Hausdorff measure of trajectories of multiparameter fractional Brownian motion. Ann. Probab. 23 (1995), no. 2, 767-775. MR-1334170

[15] D. Wu and Y. Xiao, Geometric properties of fractional Brownian sheets. J. Fourier Anal. Appl. 13 (2007), 1-37. MR-2296726

[16] Y. Xiao, Sample path properties of anisotropic Gaussian random fields. A minicourse on stochastic partial differential equations, (D Khoshnevisan and F Rassoul-Agha, editors), Lecture Notes in Math, 1962, Springer, New York (2009), 145-212. MR-2508776 


\section{Electronic Journal of Probability Electronic Communications in Probability}

\section{Advantages of publishing in EJP-ECP}

- Very high standards

- Free for authors, free for readers

- Quick publication (no backlog)

- Secure publication $\left(\mathrm{LOCKSS}^{1}\right)$

- Easy interface (EJMS²)

\section{Economical model of EJP-ECP}

- Non profit, sponsored by $\mathrm{IMS}^{3}, \mathrm{BS}^{4}$, ProjectEuclid ${ }^{5}$

- Purely electronic

\section{Help keep the journal free and vigorous}

- Donate to the IMS open access fund ${ }^{6}$ (click here to donate!)

- Submit your best articles to EJP-ECP

- Choose EJP-ECP over for-profit journals

\footnotetext{
${ }^{1}$ LOCKSS: Lots of Copies Keep Stuff Safe http://www. lockss.org/

${ }^{2}$ EJMS: Electronic Journal Management System http://www.vtex.lt/en/ejms.html

${ }^{3}$ IMS: Institute of Mathematical Statistics http://www.imstat.org/

${ }^{4}$ BS: Bernoulli Society http://www. bernoulli-society.org/

${ }^{5}$ Project Euclid: https://projecteuclid.org/

${ }^{6}$ IMS Open Access Fund: http://www.imstat.org/publications/open.htm
} 